\title{
A Unifying Approach to Interference Modeling for Wireless Networks
}

\author{
Holger Boche, Senior Member, IEEE, and Martin Schubert, Member, IEEE
}

\begin{abstract}
The paper addresses the problem of interference modeling for wireless networks. Two axiomatic approaches are known from the literature: 1) "standard interference functions" proposed by Yates in 1995, and 2) "general interference functions" proposed by the authors in their previous work. In this paper, both frameworks are thoroughly analyzed and compared. It is shown that every function from framework 1) can be expressed in terms of framework 2). This means that recent structure results for convex interference functions, which were derived for 2 ), can also be applied to 1). The results provide a bridge between the frameworks 1) and 2), which were studied separately in the literature. Also, new structure results are shown in this paper. For the example of QoS balancing, it is shown that analyzing the structure of interference functions can lead to interesting algorithmic opportunities. The results are potentially useful for the development of physical-layer aware resource allocation algorithms.
\end{abstract} tion.

Index Terms-Interference, power control, wireless communica-

\section{INTRODUCTION}

$\mathbf{I}$ NTERFERENCE modeling is important for the development of dynamic strategies for joint interference filtering and resource allocation. The performance of algorithms crucially depends on the choice of the underlying interference model.

Proposals for interference models date back to the early seventies (see, e.g., [1] and the references therein), where signal-tointerference ratios (SIR) were studied under the assumption of a simple linear interference model based on a constant link gain matrix. This linear model facilitated the application of the rich mathematical theory of nonnegative matrices, in particular the Perron-Frobenius theory, leading to many theoretical results and power control algorithms.

However, interference typically depends on the transmission powers in a nonlinear way, because adaptive receive and transmit strategies are employed to avoid or mitigate interference. Linear models are often not appropriate for the "cross-layer" problems arising from modern system architectures. While nonlinear models offer better performance, they

Manuscript received May 08, 2009; accepted December 21, 2009. Date of publication March 18, 2010; date of current version May 14, 2010. The associate editor coordinating the review of this manuscript and approving it for publication was Prof. Amir Leshem. This work was supported in part by the Bundesministerium für Bildung und Forschung (BMBF) by Grant 01SF0708 (project TEROPP).

H. Boche is with the Fraunhofer Institute for Telecommunications, HeinrichHertz-Institut, 10587 Berlin, Germany. He is also with the Fraunhofer GermanSino Lab for Mobile Communications MCI and the Technical University Berlin, 10587 Berlin, Germany.

M. Schubert is with the Fraunhofer German-Sino Lab for Mobile Communications MCI, 10587 Berlin, Germany.

Digital Object Identifier 10.1109/TSP.2010.2045415 are also more difficult to handle. So it is important to efficiently exploit the underlying mathematical structure.

Convexity was successfully exploited for the development of optimal multiuser receivers and transmitters. Many examples exist in the context of multiple-input-multiple-output (MIMO) systems and robust signal processing [2]-[8]. For example, equivalent convex reformulations exist for the downlink beamforming problem, as observed in [4]-[6]. When investigating a problem, a common approach is to first look whether the problem is convex or not.

Another useful property is monotonicity. A well-known example is the framework of standard interference functions, proposed by Yates [9] and extended in [10] and [11]. In this paper, a power control problem was solved by a fixed point iteration relying on monotonicity and scalability axioms. An application example is the downlink beamforming problem [4]-[6], [12], [13].

Monotonicity is also a key property of another axiomatic interference model that was proposed by the authors in [14]. In this work, scale invariance is proposed instead of scalability. This new axiomatic framework has also applications in power control theory, as shown in [14]. But it can also be used for the analysis of quality-of-service (QoS) regions, where the system performance can often be measured in terms of a scale-invariant indicator function (see Example 2 at the end of Section II).

These lines of research were carried out independently. To our best knowledge there has been no attempt to study convexity within the framework of standard interference functions. Also, it is not clear how standard interference functions are related to the new axiomatic approach [14]. Some connections were observed in [14], but a rigorous comparison is missing. This paper provides a unifying theory that fills this gap. The following contributions are made:

In Section II we revisit the problem of QoS balancing, which was already solved for some special cases, like beamforming or robust signal processing. Here, we formulate the problem in a very general form, based on axiomatic interference functions. By exploiting monotonicity and convexity, we show that the problem can be rewritten in an equivalent convex form. This result demonstrates that a thorough understanding of the underlying structure is very important for the design of efficient algorithms. This motivates the analytical approach presented in the following sections.

In Section III, we compare the new axiomatic framework of general (scale-invariant) interference functions with the framework of standard interference functions [9]. It is shown that any standard interference function can be expressed in terms of a scale-invariant interference function. This provides a new 
understanding of problems that were previously investigated within the framework of standard interference functions. An example is given in Section III-B, where we discuss how concavity can be exploited for solving Yates' power minimiziation problem with superlinear convergence.

In Section IV, we analyze the structure of interference functions. A new fundamental max-min decomposition is derived. Knowledge of the structure of functions is of great importance for the design of algorithms. So the results of this section provide a more complete understanding of interference functions.

In Section $\mathrm{V}$, we show that the structure results from Section IV can be transferred to standard interference functions. This is useful since many power allocation problems are naturally formulated in terms of standard interference functions. In this case, the results can be applied directly, without the need of changing from one framework to another.

Some notational conventions are: Matrices and vectors are denoted by bold capital letters and bold lowercase letters, respectively. Let $\boldsymbol{y}$ be a vector, then $y_{l}=[\boldsymbol{y}]_{l}$ is the $l$ th component. Likewise, $A_{m n}=[A]_{m n}$ is a component of the matrix $\boldsymbol{A}$. The notation $\boldsymbol{y} \geq 0$ means that $y_{l} \geq 0$ for all components $l$. Also, $\boldsymbol{y} \supsetneqq \boldsymbol{x}$ means $\boldsymbol{y} \geq \boldsymbol{x}$ and there is at least one component $l$ such that $y_{l}>x_{l}$. The set of nonnegative reals is denoted as $\mathbb{R}_{+}$. The set of positive reals is denoted as $\mathbb{R}_{++}$. The notation $\exp (\boldsymbol{y})$ and $\log (\boldsymbol{y})$ means component-wise exponential and logarithm, respectively. Let $\mathcal{P} \subseteq \mathbb{R}_{+}^{n}$, then $\log \mathcal{P}=\left\{\boldsymbol{s}=\log (\boldsymbol{p}): \boldsymbol{p} \in \mathcal{P} \cap \mathbb{R}_{++}^{n}\right\}$.

\section{INTERFERENCE MODELING, MotivATING EXAMPLES, AND FIRST RESULTS}

Consider a coupled multiuser system with resources $\boldsymbol{r}=$ $\left[r_{1}, r_{2}, \ldots, r_{K}\right]^{T} \in \mathbb{R}_{+}^{K}$, where $K \geq 2$ is an arbitrary dimension. Also, let $\mathcal{K}=\{1,2, \ldots, K\}$. We use the concept of interference functions to model the impact of $\boldsymbol{r}$ on certain system state measures, like interference. We begin with the definition, then examples will be discussed.

Definition 1: Let $\mathcal{I}: \mathbb{R}_{+}^{K} \mapsto \mathbb{R}_{+}$. We say that $\mathcal{I}$ is a general interference function (or simply interference function) if the following axioms are fulfilled:
A1(positivity)
There is an $\boldsymbol{r}>0$ such that $\mathcal{I}(\boldsymbol{r})>0$
A2 (scale invariance)
$\mathcal{I}(\alpha \boldsymbol{r})=\alpha \mathcal{I}(\boldsymbol{r})$ for all $\alpha>0$
A3 (monotonicity)
$\mathcal{I}(\boldsymbol{r}) \geq \mathcal{I}\left(\boldsymbol{r}^{\prime}\right)$ if $\boldsymbol{r} \geq \boldsymbol{r}^{\prime}$

The axioms A1, A2, A3 were proposed in [14] for modeling interference in wireless networks, hence, the name "interference function." The connection with Yates' well-known framework of standard interference functions [9] will be studied in Section III.

The following two examples show possible applications of the framework A1, A2, A3. Example 1 is on classical interference modeling. Example 2 shows that interference functions also provide a more abstract and general way of modeling dependencies between users.

1) Example 1: Consider a wireless multiuser channel with $K_{u}$ users, with index set $\mathcal{K}_{u}=\left\{1,2, \ldots, K_{u}\right\}$. The users' transmission powers are collected in a vector $\boldsymbol{p}=\left[p_{1}, p_{2}, \ldots, p_{K_{u}}\right]^{T} \in \mathbb{R}_{+}^{K_{u}}$. Each link is corrupted by noise with power $\sigma_{n}^{2}>0$. We define the extended power vector

$$
\underline{\boldsymbol{p}}=\left[\begin{array}{c}
\boldsymbol{p} \\
\sigma_{n}^{2}
\end{array}\right]=\left[p_{1}, \ldots, p_{K_{u}}, \sigma_{n}^{2}\right]^{T} .
$$

Let $\boldsymbol{v}_{k} \in \mathbb{R}_{+}^{K_{u}}$ be a vector of interference coupling coefficients that determines the power crosstalk in the system, then the resulting interference at user $k$ is

$$
\mathcal{I}_{k}(\underline{\boldsymbol{p}})=\boldsymbol{v}_{k}^{T} \boldsymbol{p}+\sigma_{n}^{2} .
$$

In the Appendix I-A we show how the model (2) can be extended by multiuser beamforming, which leads to a nonlinear interference function.

2) Example 2: Consider the interference functions from Example 1. The resulting signal-to-interference-plus-noise ratios (SINR) are

$$
\operatorname{SINR}(\boldsymbol{p})=\frac{p_{k}}{\mathcal{I}_{k}(\underline{\boldsymbol{p}})}, \quad k \in \mathcal{K}_{u} .
$$

Let $\gamma \in \mathbb{R}_{+}^{K_{u}}$ be a vector of SINR targets, and $\mathcal{P} \subset \mathbb{R}_{+}^{K_{u}}$ a set of possible transmission powers, then

$$
C(\gamma)=\inf _{\boldsymbol{p} \in \mathcal{P}}\left(\max _{k \in \mathcal{K}} \frac{\gamma_{k} \cdot \mathcal{I}_{k}(\underline{\boldsymbol{p}})}{p_{k}}\right)
$$

is an indicator for feasibility [14]. That is, the targets $\gamma$ are feasible if and only if $C(\boldsymbol{\gamma}) \leq 1$. The feasible SINR region is defined as

$$
\mathcal{S}=\{\boldsymbol{\gamma} \geq 0: C(\gamma) \leq 1\} .
$$

The function $C(\boldsymbol{\gamma})$ fulfills the axioms $\mathrm{A} 1, \mathrm{~A} 2, \mathrm{~A} 3$, so it is an interference function.

This demonstrates that interference functions do not just occur in the classical power control context. They rather provide a general framework, which is useful for modeling different kinds of dependencies between users. Further motivation for using the framework A1, A2, A3 will be provided in Section III. These three axioms provide the basis for what we understand as "interference functions".

General properties of interference functions will be studied in Section IV. Most other parts of the paper will focus on the particular class of strictly monotone interference functions, which will be introduced in the next subsection.

\section{A. Strict Monotonicity and Convexity}

Parts of this paper are focused on the particular case of power control, where the interference function $\mathcal{I}$ depends on the $\left(K_{u}+\right.$ 1)-dimensional power vector (1). In order to model the impact of noise adequately, we require additional strict monotonicity

$$
\begin{aligned}
& \text { A4 (strict monotonicity) } \\
& \quad \mathcal{I}(\underline{\boldsymbol{p}})>\mathcal{I}\left(\underline{\boldsymbol{p}}^{\prime}\right) \text { if } \boldsymbol{p} \geq \boldsymbol{p}^{\prime} \text { and } p_{K_{u}+1}>p_{K_{u}+1}^{\prime} .
\end{aligned}
$$

Axiom A4 is explained most easily by Example 1. The interference function (2) fulfills A4. Also the interference function resulting from beamforming (Appendix I-A) fulfills A4. 
Axiom A4 is a key property that is important for the analysis of wireless systems with receiver noise and power constraints. If $p_{K_{u}+1}>0$, then A4 ensures that $\mathcal{I}(\underline{\boldsymbol{p}})>0$. This will be exploited in Section II-B. In Section III, it will be shown that A4 is the property that links the framework A1, A2, A3 with the framework of standard interference functions [9].

Besides monotonicity, there are other properties which can be exploited, if available. In the following we will also study convex and concave interference functions, which are defined on a convex domain $\mathcal{P}$. Examples of concave interference functions are again (2) and the beamforming interference from Appendix I-A.

Finally, we will study logarithmically convex (log-convex) interference functions. The following definition is based on a change of variable $s=\log p$.

Definition 2: We say that $\mathcal{I}: \mathcal{P} \mapsto \mathbb{R}_{+}$is a log-convex interference function if $\mathrm{A} 1, \mathrm{~A} 2, \mathrm{~A} 3$ are fulfilled and in addition $\log \mathcal{I}(\exp \{\boldsymbol{s}\})$ is convex on $\log \mathcal{P}$.

It was shown in [15] that every convex interference function is a log-convex interference function, however the converse is not true. Thus the class of convex interference functions is contained in the broader class of log-convex interference functions. Log-convex interference functions offer interesting analytical possibilities similar to the convex case, while being less demanding. For certain nonconvex problems the logarithmic transformation leads to an equivalent convex reformulation. For an overview, see, e.g., [16] and the references therein.

\section{B. The Quality-Of-Service (QoS) Balancing Problem-Revisited}

We begin with an example that demonstrates the importance of exploiting strict monotonicity. Consider the common problem of achieving QoS targets $\boldsymbol{u}^{\min }=\left[u_{1}^{\min }, \ldots, u_{K_{u}}^{\min }\right]^{T}>$ 0 with minimum use of transmission powers.

$$
\min _{\boldsymbol{p} \in \mathcal{P}} \sum_{l \in \mathcal{K}_{u}} p_{l} \quad \text { s.t. } \quad u_{k}(\boldsymbol{p}) \geq u_{k}^{\min } \text { for all } k \in \mathcal{K}_{u} .
$$

Here, $u_{k}(\boldsymbol{p})$ is defined as an abstract performance measure depending on the SINR via a strictly monotone and continuous function $\phi: \mathbb{R}_{++} \mapsto \mathbb{R}$.

$$
u_{k}(\boldsymbol{p})=\phi_{k}\left(\operatorname{SINR}_{k}(\boldsymbol{p})\right), \quad k \in \mathcal{K}_{u}
$$

Problem (7) is closely connected with the max-min formulation

$$
\max _{\boldsymbol{p} \in \mathcal{P}}\left(\min _{k \in \mathcal{K}_{u}} \frac{u_{k}(\boldsymbol{p})}{u_{k}^{\min }}\right) .
$$

If the power set $\mathcal{P}$ is bounded, then both problems (7) and (9) are equivalent in a sense that the solution of one problem can be found indirectly via a bisection strategy involving the other problem. If $\boldsymbol{u}^{\min }$ is a point on the boundary of the feasible set, then both problems yield the same optimizer. This is the unique fixed point described by Yates [9]. So both problems can be comprehended under the name "QoS balancing."

We will now focus on problem (7) which is more convenient to analyze. Let $\phi^{[-1]}$ be the inverse function of $\phi$, then $\gamma_{k}=$ $\phi_{k}^{[-1]}\left(u_{k}^{\min }\right)$ is the minimum SINR level needed by the $k$ th user to achieve some feasible target $u_{k}^{\min }$. So the optimizer of (7) is obtained by solving the SINR balancing problem

$$
\min _{\boldsymbol{p} \in \mathcal{P}} \sum_{\boldsymbol{l} \in \mathcal{K}_{u}} p_{\boldsymbol{l}} \quad \text { s.t. } \quad \frac{p_{k}}{\mathcal{I}_{k}(\underline{\boldsymbol{p}})} \geq \gamma_{k}, \text { for all } k \in \mathcal{K}_{u} .
$$

Under the assumption of strict monotonicity A4, this problem can be solved by a globally convergent fixed point iteration, which will be discussed later in Section III-A. The iteration has linear convergence [10], [17], regardless of the actual choice of $\mathcal{I}_{k}$.

More efficient solutions are available if the interference functions $\mathcal{I}_{k}$ are convex. We can rewrite (10) in equivalent form

$$
\min _{\boldsymbol{p} \in \mathcal{P}} \sum_{l \in \mathcal{K}_{u}} p_{l} \quad \text { s.t. } \quad \gamma_{k} \mathcal{I}_{k}(\underline{\boldsymbol{p}})-p_{k} \leq 0, \text { for all } k \in \mathcal{K}_{u}
$$

If the power set $\mathcal{P} \subseteq \mathbb{R}_{++}^{K_{u}}$ is convex, which is typically fulfilled, then (11) is a convex optimization problem. Property A4 ensures the existence of a nontrivial solution, provided that the targets $\gamma_{k}$ are feasible.

Next, consider the case where $\mathcal{I}_{k}$ is strictly monotonic and concave. An example is the beamforming problem in Appendix I-A, with either individual power constraints or a total power constraint. Then, problem (11) is nonconvex because the constraints are concave, but not convex.

This observation is in line with the literature on multiuser beamforming [4]-[6], where it was observed that the corresponding problem is nonconvex in its direct form, but equivalent convex reformulations exist. So an interesting question is: does an equivalent convex reformulation also exist for the more general problem (11), which is only based on the axiomatic framwork with the additional assumptions of strict monotonicity and concavity? This is answered by the following theorem.

Theorem 1: Let $\mathcal{I}_{1}, \ldots, \mathcal{I}_{K_{u}}$ be concave and strictly monotonic interference functions, then the optimizer of problem (11) is equivalently obtained by the convex problem

$$
\max _{\boldsymbol{p} \in \mathcal{P}} \sum_{l \in \mathcal{K}_{u}} p_{\boldsymbol{l}} \quad \text { s.t. } \quad p_{k}-\gamma_{k} \mathcal{I}_{k}(\underline{\boldsymbol{p}}) \leq 0, \quad \forall k \in \mathcal{K}_{u} .
$$

Proof: First, we observe that (11) is feasible if and only if (12) is feasible. Assume that (12) is feasible. Because of strict monotonicity A4 there must exist a vector $\boldsymbol{p}^{*}>0$ such that all inequalities in (12) are fulfilled with equality. This implies feasibility of (11). The converse is shown likewise.

Let $\mathcal{I}(\boldsymbol{p})=\left[\mathcal{I}_{1}(\boldsymbol{p}), \ldots, \mathcal{I}_{K_{u}}(\boldsymbol{p})\right]^{T}$. The vector $\boldsymbol{p}^{*}$ is the unique fixed point that satisfies $\boldsymbol{p}^{*}=\operatorname{diag}(\boldsymbol{\gamma}) \mathcal{I}\left(\underline{p}^{*}\right)$. This is the optimizer of (11), as shown in [9]. The same fixed point $\boldsymbol{p}^{*}$ is achieved by (12). This can easily be shown by contradiction. If there would exist a $k_{0}$ such that the optimizer $\boldsymbol{p}^{*}$ fulfills $p_{k_{0}}^{*}-\gamma_{k} \mathcal{I}_{k_{0}}\left(\underline{\boldsymbol{p}}^{*}\right)<0$, then we could increase $p_{k_{0}}^{*}$ without violating the constraints. This would mean that we could achieve a point larger than the global maximum. Therefore, (12) yields the fixed point $\boldsymbol{p}^{*}$ which also solves (11).

Problem (12) is convex and can be solved by applying standard solutions from convex optimization theory. This also sheds some new light on the problem of multiuser beamforming [4]-[6], [12], [13], which is contained as a special case. It turns 
out that this problem has a generic convex form (12) which can be used as a basis for the development of algorithms.

However, general purpose solvers can be inefficient. A better performance is typically achieved by exploiting the analytical structure of the problem at hand. Later, in Section III-B we will discuss how the properties A1 to A4 can be exploited for the design of a generic algorithm with superlinear convergence.

Next, consider the class of log-convex interference functions (see Definition 2). Examples are worst-case interference designs used in the context of robust optimization (see, e.g., [7] and [8]). Every convex interference function is a log-convex interference functions, as mentioned before, so the following result also applies to convex interference functions.

Theorem 2: Let $\mathcal{I}_{1}, \ldots, \mathcal{I}_{K_{u}}$ be log-convex and strictly monotonic interference functions, then the optimizer $\boldsymbol{p}^{*}$ of problem (11) is obtained as $\boldsymbol{p}^{*}=\exp \boldsymbol{s}^{*}$, where $\boldsymbol{s}^{*}$ is the optimizer of

$$
\begin{aligned}
\min _{\boldsymbol{s} \in \log \mathcal{P}} \sum_{l \in \mathcal{K}_{u}} s_{l} & \text { s.t. } \quad \log \gamma_{k} \\
& +\log \mathcal{I}_{k}(\exp \underline{\boldsymbol{s}})-s_{k} \leq 0 \quad \forall k \in \mathcal{K}_{u} .
\end{aligned}
$$

Proof: Exploiting the strict monotonicity of the logarithm, we can rewrite the constraints in (11) as

$$
\log \gamma_{k} \mathcal{I}_{k}(\underline{\boldsymbol{p}})-\log p_{k} \leq 0
$$

Introducing the change of variable $s=\log \boldsymbol{p}$, this can be rewritten as

$$
\log \gamma_{k}+\log \mathcal{I}_{k}(\exp \underline{\boldsymbol{s}})-s_{k} \leq 0
$$

Using the same argumentation as in the proof of Theorem 1, it follows from A4 that the constraints in (13) are fulfilled with equality in the optimum, so the optimizer $s^{*}$ is the unique fixed point in the transformed domain.

The constraints in (13) are convex because $\log \mathcal{I}_{k}(\exp \underline{\boldsymbol{s}})$ is convex by definition. Also, the domain $\log \mathcal{P}$ is convex if $\mathcal{P} \subseteq$ $\mathbb{R}_{++}^{K_{u}}$ is a downward-comprehensive convex set. Comprehensiveness is defined as below. It is fulfilled for many cases of interest (e.g., unconstrained powers, per-user power constraints, sum-power constraint).

Definition 3: A set $\mathcal{V} \subset \mathbb{R}_{++}^{K_{u}}$ is said to be upward-comprehensive if for all $\boldsymbol{w} \in \mathcal{V}$ and $\boldsymbol{w}^{\prime} \in \mathbb{R}_{++}^{K_{u}}$

$$
\boldsymbol{w}^{\prime} \geq \boldsymbol{w} \quad \Rightarrow \quad \boldsymbol{w}^{\prime} \in \mathcal{V}
$$

If the inequality is reversed, then $\mathcal{V}$ is said to be downwardcomprehensive.

\section{COMPARISON BETWEen GENERAL AND STANDARD INTERFERENCE FUNCTIONS}

In the previous section we have shown that the general axiomatic framework A1, A2, A3, in combination with strict monotonicity A4, can be used to find equivalent convex reformulations for certain resource allocation problems which are nonconvex in its original form.
In this section we will address the following questions:

- Yates' framework of standard interference functions [9] is an established interference model, so what is the motivation for the framework A1, A2, A3?

- Both axiomatic frameworks are similar, so how exactly are they connected?

We begin by introducing standard interference functions.

\section{A. Standard Interference Functions}

In [9], Yates introduced an axiomatic framework of standard interference functions, with extensions in [10], [11]. This framework was motivated by the SINR balancing problem discussed in Section II-B.

Definition 4: A function $Y: \mathbb{R}_{+}^{K_{u}} \mapsto \mathbb{R}_{++}$is said to be a standard interference function if the following axioms are fulfilled:

$$
\begin{array}{ll}
\text { Y1 ( positivity) } & Y(\boldsymbol{p})>0 \text { for all } \boldsymbol{p} \in \mathbb{R}_{+}^{K_{u}} \\
\text { Y2 (scalability) } & \alpha Y(\boldsymbol{p})>Y(\alpha \boldsymbol{p}) \text { for all } \alpha>1 \\
\text { Y3 (monotonicity) } & Y(\boldsymbol{p}) \geq Y\left(\boldsymbol{p}^{\prime}\right) \text { if } \boldsymbol{p} \geq \boldsymbol{p}^{\prime}
\end{array}
$$

A simple example for a standard interference function is the linear interference model (2), which can be written as $Y_{k}(\boldsymbol{p})=$ $\boldsymbol{v}_{k}^{T} \boldsymbol{p}+\sigma_{n}^{2}$. Other examples can be found, for example, in the context of multiuser beamforming [4]-[6], [12], [13], CDMA [18], [19], base station assignment [20], [21], and robust designs [7], [8].

If the targets $\gamma_{1}, \ldots, \gamma_{K_{u}}$ are feasible, then the fixed point iteration

$$
p_{k}^{(n+1)}=\gamma_{k} Y_{k}\left(\boldsymbol{p}^{(n)}\right), \quad \forall k \in \mathcal{K}_{u}, \quad \boldsymbol{p}^{(0)} \in \mathbb{R}_{+}^{K_{u}}
$$

converges globally to the unique optimizer of the power minimization problem (10), as shown in [9]. Convergence properties of this iteration were investigated in [10], [11], and [17].

\section{B. Exploiting the Structure of Interference Functions}

In Section II-B we have used the framework A1, A2, A3, plus strict monotonicity A4, although the same conclusions could have been achieved by using standard interference functions characterized by $\mathrm{Y} 1, \mathrm{Y} 2, \mathrm{Y} 3$. We will now discuss some reasons for favoring A1, A2, A3 over standard interference functions.

One advantage is its generality. It will be shown in the following Section III-C that any standard interference function can be expressed by an equivalent interference function fulfilling $\mathrm{A} 1, \mathrm{~A} 2, \mathrm{~A} 3$. Thus, any problem involving standard interference functions can be reformulated in terms of the framework A1, A2, A3. Moreover, the framework is suitable for modeling various other kinds of multiuser performance measures, including indicator functions for feasibility, and combinations of user utilities (see, e.g., Example 2 in Section II and [14]-[16], [22]). This generalized notion of interference abstracts away from its original physical meaning. It contributes to a better understanding of multiuser QoS/utility sets, and also provides a bridge to gametheoretical strategies (see, e.g., [23]).

Another benefit of the framework A1, A2, A3 is its amenability to structural analysis. Any interference function can be expressed as a maximum or minimum of certain 
elementary interference functions [22]. More structure results are available if the interference functions has additional properties, like convexity, concavity [15], or logarithmic convexity [16]. Knowledge about this structure can lead to interesting analytical opportunities. This will now be demonstrated by an example.

Consider the QoS balancing problem from Section II-B with concave and strictly monotonic interference functions. The problem can be solved with superlinear convergence if we exploit the structure results shown in [15]. In particular, we use the following lemma, which holds for arbitrary concave interference function fulfilling A1, A2, A3.

Lemma 1: An interference function $\mathcal{I}$ is concave if and only if there exists a nonempty upward-comprehensive closed convex set $\mathcal{V}_{\mathcal{I}} \subset \mathbb{R}_{+}^{K}$ such that

$$
\mathcal{I}(\boldsymbol{p})=\min _{\boldsymbol{v} \in \mathcal{V}_{\mathcal{I}}} \sum_{k \in \mathcal{K}} v_{k} p_{k}, \quad \text { for all } \boldsymbol{p}>0 .
$$

This result opens up new perspectives for a more general understanding of interference functions. For example, (15) can be regarded as the optimum of a weighted cost minimization over some strategy set $\mathcal{V}_{\mathcal{I}}$, with weighting factors $p_{k}$. One can imagine many other possible interpretations of the variable $\boldsymbol{p}$. However, in order to keep the discussion simple, we will confine ourselves to the aformentioned QoS balancing example. The following discussion shows that Lemma 1 is useful for developing an algorithmic solution for problem (11).

Consider the $\left(K_{u}+1\right)$-dimensional vector $\boldsymbol{p}$ defined by (1). The $K_{u}$ interference functions $\mathcal{I}_{1}, \ldots, \mathcal{I}_{K_{u}}$ are associated with coefficient sets $\mathcal{V}_{1}, \ldots, \mathcal{V}_{K_{u}} \subset \mathbb{R}_{+}^{K_{u}+1}$. From Lemma 1 we know that for any given $\boldsymbol{p}$, we have

$$
\mathcal{I}_{k}(\underline{\boldsymbol{p}})=\min _{\boldsymbol{v} \in \mathcal{V}_{k}}\left(\sum_{l \in \mathcal{K}_{u}} v_{l} p_{l}+\sigma_{n}^{2} \cdot v_{K_{u}+1}\right) .
$$

The coefficients $v_{1}, \ldots, v_{K_{u}}$ determine the interference coupling between the users, while $v_{K_{u}+1}$ amplifies the noise. This is a typical structure for many interference scenarios involving adaptive receive or transmit strategies. An example is the aforementioned beamforming problem (see Appendix I-A).

Let $\boldsymbol{v}(z)$ be the coupling vector resulting from some unspecified receive strategy $z$, chosen from a compact set $\mathcal{Z}_{k}$. Then, (16) can be rewritten as

$$
\mathcal{I}_{k}(\underline{\boldsymbol{p}})=\min _{z \in \mathcal{Z}_{k}}\left(\sum_{l \in \mathcal{K}_{u}} v_{l}(z) p_{l}+\sigma_{n}^{2} \cdot v_{K_{u}+1}(z)\right) .
$$

Here, $z$ is an arbitrary system parameter that determines the interference coupling. A special case is the beamforming example from Appendix I-A. Each beamformer is constrained to a compact set $\mathcal{Z}_{k}$, which is typically the unit sphere. ${ }^{1}$ It was shown in [15] that this leads to a convex comprehensive set of coupling vectors $\mathcal{V}_{k}$, each of which is associated with a concave interference function (16).

Lemma 1 shows the converse direction: for any concave interference function there is a representation (17). Thus, we can

\footnotetext{
${ }^{1}$ Arbitrary constraints can be imposed on the beamformers, like the shaping constraints in [5]. We only require that $\mathcal{Z}_{k}$ is compact, to ensure that the minimum exists.
}

use the results [17], where an iterative "Newton-type" algorithm with superlinear convergence was proposed for solving problem (11). While this algorithm was only derived for interference functions of the form (17), an immediate consequence of Lemma 1 is that such an iteration also exists for arbitrary concave interference functions. The details of this iteration can be found in [17]. To summarize: the QoS balancing problem from Section II-B can be solved with superlinear convergence if the underlying interference functions are concave or convex.

This example shows that analyzing the structure of A1, A2, A3 can be very useful for the development of algorithms. However, no corresponding results exist for standard interference functions. It will be one contribution of this paper (Section V) to show that certain structure results like Lemma 1 can be transfered to standard interference functions. We will also derive new structure results in Section IV, which complement the results already shown in [15].

It remains to answer the second question: How are these results connected with standard interference functions? The structure results for convex/concave interference functions were derived for the framework A1, A2, A3. It is not obvious whether these results can be transfered to the framework Y1,Y2,Y3 or not. Later, in Section $\mathrm{V}$ we will show that the results indeed extend to standard interference functions. To this end, we first need to establish a link between both frameworks. This will be done in the remainder of this section.

\section{Comparison Between Both Axiomatic Frameworks}

When comparing the two axiomatic frameworks in Definitions 1 and 4, it can be observed that the main difference is between Y2 and A2. In order to establish a link between both frameworks, we introduce the following definition.

Definition 5: A function $Y: \mathbb{R}_{+}^{K_{u}} \mapsto \mathbb{R}_{++}$is said to be a weakly standard interference function if the following axiom Y2' is fulfilled together with Y1 (positivity) and Y3 (monotonicity).

\section{$\mathbf{Y} \mathbf{2}^{\prime}$ (weak scalability) $\quad \alpha Y(\boldsymbol{p}) \geq Y(\alpha \boldsymbol{p})$ for all $\alpha \geq 1$.}

The following theorem shows how general interference functions $\mathcal{I}$ and standard interference functions $Y$ are related. To this end, we introduce the power set

$$
\underline{\mathcal{P}}=\left\{\underline{\boldsymbol{p}}=\left[\begin{array}{c}
\boldsymbol{p} \\
\underline{p}_{K_{u}+1}
\end{array}\right]: \boldsymbol{p} \in \mathbb{R}_{+}^{K_{u}}, \underline{p}_{K_{u}+1} \in \mathbb{R}_{++}\right\} .
$$

In a power control context, $\boldsymbol{p}$ is a vector of transmission powers and $\underline{p}_{K_{u}+1}$ is the noise power. For notational convenience, we define $\mathcal{I}(\underline{\boldsymbol{p}})=\mathcal{I}\left(\boldsymbol{p}, \underline{p}_{K_{u}+1}\right)$.

Theorem 3:

1) Let $Y: \mathbb{R}_{+}^{K_{u}} \mapsto \mathbb{R}_{++}$be a weakly standard interference function, then the extended function

$$
\begin{aligned}
\mathcal{I}_{Y}(\underline{\boldsymbol{p}}) & :=\mathcal{I}_{Y}\left(\boldsymbol{p}, \underline{p}_{K_{u}+1}\right) \\
& =\underline{p}_{K_{u}+1} \cdot Y\left(\frac{p_{1}}{\underline{p}_{K_{u}+1}}, \ldots, \frac{p_{K_{u}}}{\underline{p}_{K_{u}+1}}\right)
\end{aligned}
$$

is a general interference function on $\underline{\mathcal{P}}$. We have

$$
Y(\boldsymbol{p})=\mathcal{I}_{Y}(\boldsymbol{p}, 1) \quad \text { for all } \boldsymbol{p} \geq 0 \text {. }
$$


2) Let $\mathcal{I}: \mathbb{R}_{+}^{K_{u}+1} \mapsto \mathbb{R}_{+}$be a general interference function, then for any given $\underline{p}_{K_{u}+1}>0$, the reduced function

$$
Y_{\mathcal{I}}(\boldsymbol{p}):=\mathcal{I}\left(p_{1}, \ldots, p_{K_{u}}, \underline{p}_{K_{u}+1}\right)
$$

is a weakly standard interference function on $\mathbb{R}_{++}^{K_{u}}$.

3) Let $\mathcal{I}_{Y}$ be defined as in (19). Then $Y$ is a standard interference function if and only if $\mathcal{I}_{Y}$ fulfills A1, A2, A3, and for all $\boldsymbol{p} \in \mathbb{R}_{+}^{K_{u}}$, the function $\mathcal{I}_{Y}\left(\boldsymbol{p}, \underline{p}_{K_{u}+1}\right)$ fulfills A4 (strict monotonicity with respect to $\underline{p}_{K_{u}+1}>0$ ).

Proof: We begin by proving 1). Axiom A1 is fulfilled because $\mathcal{I}_{Y}(\boldsymbol{p})>0$ for all $\boldsymbol{p} \in \underline{\mathcal{P}}$. Axiom A2 (scale invariance) is fulfilled because for all $\bar{\lambda}>0$

$$
\begin{aligned}
\mathcal{I}_{Y}(\lambda \underline{\boldsymbol{p}}) & =\lambda \cdot p_{K_{u}+1} \cdot Y\left(\frac{\lambda p_{1}}{\lambda p_{K_{u}+1}}, \ldots, \frac{\lambda p_{K_{u}}}{\lambda p_{K_{u}+1}}\right) \\
& =\lambda \mathcal{I}_{Y}(\underline{\boldsymbol{p}}) .
\end{aligned}
$$

It remains to show A3 (monotonicity). Consider two arbitrary vectors $\boldsymbol{p}^{(1)}, \boldsymbol{p}^{(2)} \in \underline{\mathcal{P}}$ such that $\boldsymbol{p}^{(1)} \geq \boldsymbol{p}^{(2)}$. With $\tilde{\lambda}=p_{K_{u}+1}^{(1)} / p_{K_{u}+1}^{(2)} \geq 1$, we have

$$
\begin{aligned}
\mathcal{I}_{Y}\left(\underline{\boldsymbol{p}}^{(2)}\right) & =p_{K_{u}+1}^{(2)} \cdot Y\left(\frac{p_{1}^{(2)}}{p_{K_{u}+1}^{(2)}}, \ldots, \frac{p_{K_{u}}^{(2)}}{p_{K_{u}+1}^{(2)}}\right) \\
& =p_{K_{u}+1}^{(2)} \cdot Y\left(\tilde{\lambda} \frac{p_{1}^{(2)}}{p_{K_{u}+1}^{(1)}}, \ldots, \tilde{\lambda} \frac{p_{K_{u}}^{(2)}}{p_{K_{u}+1}^{(1)}}\right) \\
& \leq p_{K_{u}+1}^{(2)} \cdot Y\left(\tilde{\lambda} \frac{p_{1}^{(1)}}{p_{K_{u}+1}^{(1)}}, \ldots, \tilde{\lambda} \frac{p_{K_{u}}^{(1)}}{p_{K_{u}+1}^{(1)}}\right) \\
& \leq p_{K_{u}+1}^{(2)} \cdot \tilde{\lambda} \cdot Y\left(\frac{p_{1}^{(1)}}{p_{K_{u}+1}^{(1)}}, \ldots, \frac{p_{K_{u}}^{(1)}}{p_{K_{u}+1}^{(1)}}\right) \\
& =p_{K_{u}+1}^{(1)} \cdot Y\left(\frac{p_{1}^{(1)}}{p_{K_{u}+1}^{(1)}}, \ldots, \frac{p_{K_{u}}^{(1)}}{p_{K_{u}+1}^{(1)}}\right) \\
& =\mathcal{I}_{Y}\left(\underline{\boldsymbol{p}}^{(1)}\right) .
\end{aligned}
$$

The first inequality follows from $Y 3$ (monotonicity) and the second from $Y 2^{\prime}$ (weak scalability).

We now prove 2). Axiom Y3 follows directly from A3. Axiom Y1 holds on $\mathbb{R}_{++}^{K_{u}}$ because $\mathcal{I}(\underline{p})>0$ for all $\underline{p}>0$. This is a consequence of $\mathrm{A} 1$, as shown in [14]. Axiom $\mathrm{Y} 2$ ' follows from

$$
\begin{aligned}
& Y(\alpha \boldsymbol{p})= \mathcal{I}\left(\alpha \boldsymbol{p}, \underline{p}_{K_{u}+1}\right) \leq \mathcal{I}\left(\alpha \boldsymbol{p}, \alpha \underline{p}_{K_{u}+1}\right) \\
&=\alpha \mathcal{I}\left(\boldsymbol{p}, \underline{p}_{K_{u}+1}\right)=\alpha Y(\boldsymbol{p}) .
\end{aligned}
$$

Note that this inequality need not be strict because we did not made any assumption on whether $\mathcal{I}$ depends on $\underline{p}_{K_{u}+1}$ or not.

We now prove 3). Let $Y$ be standard. From 1) we know that $\mathcal{I}_{Y}(\boldsymbol{p})$ fulfills A1, A2, A3. We now show strict monotonicity. For arbitrary $\underline{\boldsymbol{p}}^{(1)}, \underline{\boldsymbol{p}}^{(2)} \in \underline{\mathcal{P}}$, with $\boldsymbol{p}^{(1)}=\boldsymbol{p}^{(2)}$ and $\tilde{\lambda}=p_{K_{u}+1}^{(1)} / p_{K_{u}+1}^{(2)}>1$ the second inequality (22) is strict. This follows from Y2 (which holds for $\alpha>1$ because of continuity). Thus, $\mathcal{I}_{Y}(\boldsymbol{p})$ is strictly monotone with respect to the component $p_{K_{u}+1}$. Conversely, let $\mathcal{I}_{Y}$ be strictly monotone and A1, A2, A3 are fulfilled. Then

$$
Y(\lambda \boldsymbol{p})=\mathcal{I}_{Y}(\lambda \boldsymbol{p}, 1)=\lambda \mathcal{I}\left(\boldsymbol{p}, \frac{1}{\lambda}\right)<\lambda \mathcal{I}_{Y}(\boldsymbol{p}, 1)=\lambda Y(\boldsymbol{p})
$$

for all $\alpha>0$, thus Y2 holds. Property Y3 follows directly from A3. Finally, we show Y1 by contradiction. Suppose that there exists a $\boldsymbol{p} \in \underline{\mathcal{P}}$ such that $Y(\boldsymbol{p})=0$. Strict monotonicity of $\mathcal{I}$ implies

$$
0=Y(\boldsymbol{p})=\mathcal{I}(\underline{\boldsymbol{p}})>\mathcal{I}(\alpha \underline{\boldsymbol{p}})=\alpha \mathcal{I}(\underline{\boldsymbol{p}}), \quad 0<\alpha<1 .
$$

Letting $\alpha \rightarrow 0$ we obtain a contradiction, thus proving $\mathrm{Y} 1$.

Theorem 3 shows that any standard interference function has an equivalent representation in terms of the extended model. The next corollary is an immediate application of Theorem 3.

Corollary 1: Any (weakly) standard interference function $Y$ is continuous on $\mathbb{R}_{++}^{K_{u}}$.

Proof: This is a direct consequence of Theorem 3, which states that, for any $Y$ exists a general interference function $\mathcal{I}_{Y}$ such that $Y(\boldsymbol{p})=\mathcal{I}_{Y}(\boldsymbol{p}, 1)$ for all $\boldsymbol{p}$. Any general interference function is continuous on $\mathbb{R}_{++}^{K}$, as shown in [14].

This property was implicitly assumed in [9]. Corollary 1 justifies this assumption in hindsight. The proof in [9] is only rigorous with the continuity stated by Corollary 1 . Note that Corollary 1 only states continuity on $\mathbb{R}_{++}^{K_{u}}$ not on $\mathbb{R}_{+}^{K_{u}}$. This is sufficient for the fixed point iteration [9] because the limit of this iteration is always strictly positive. Continuity on $\mathbb{R}_{+}^{K_{u}}$ will be shown in the following Section III-D.

\section{Continuation on the Boundary}

Parts of this paper build on our previous work [15], [16], [22], where properties of interference functions $\mathcal{I}$ were analyzed. Some of these results, like the aforementioned continuity, were only shown for a restricted domain $\mathbb{R}_{++}^{K}$ instead of $\mathbb{R}_{+}^{K}$. This technical assumption was made for the sake of mathematical tractability. By requiring $\boldsymbol{p}>0$ we ensure $\mathcal{I}(\boldsymbol{p})>0$. This is sometimes needed to avoid singularities, e.g., when dealing with signal-to-interference ratios $p_{k} / \mathcal{I}_{k}(\boldsymbol{p})$.

Sometimes, the assumption $\boldsymbol{p}>0$ is not very restrictive, like in the case of the SINR balancing problem (10), which has a strictly positive solution. However, there are many other resource allocation problems that require $p_{k}=0$, meaning that user $k$ is inactive. One way of handling this case is to let $p_{k}$ tend to zero.

In the remainder of this section, we will study the consequences that this axiomatic approach has on the interference functions. Assume that $\mathcal{I}(\boldsymbol{p})$ is defined on $\mathbb{R}_{++}^{K}$. Let $\boldsymbol{p}^{(n)} \in \mathbb{R}_{++}^{K}$ be an arbitrary sequence with $\operatorname{limit} \lim _{n} \rightarrow \infty \boldsymbol{p}^{(n)}=\boldsymbol{p} \in \mathbb{R}_{+}^{K}$. We define the continuation

$$
\mathcal{I}^{c}(\boldsymbol{p})=\lim _{n \rightarrow \infty} \mathcal{I}\left(\boldsymbol{p}^{(n)}\right) .
$$

The following theorem states that for any interference function, the properties A1, A2, A3 are preserved on the boundary of the power set, where powers are zero.

Theorem 4: Let $\mathcal{I}$ be an arbitrary interference function defined on $\mathbb{R}_{++}^{K}$. Then, the continuation $\mathcal{I}^{c}(\boldsymbol{p})$ defined on $\mathbb{R}_{+}^{K}$ fulfills the axioms A1, A2, A3. 
Proof: We need the lemmas shown in Appendix I-B. Axiom A3 (monotonicity) follows from Lemma 9. Axiom A2 (scale invariance) follows from Lemma 6 . Axiom A1 is also fulfilled since $\mathcal{I}(\boldsymbol{p})=\mathcal{I}^{c}(\boldsymbol{p})$ for all $\boldsymbol{p} \in \mathbb{R}_{++}^{K}$.

We can use this continuation to extend results that were previously shown for $\mathbb{R}_{++}^{K}$. As an example, consider Corollary 1 , which states continuity on $\mathbb{R}_{++}^{K}$. This is now extended to $\mathbb{R}_{+}^{K}$ by the following theorem. More examples will follow.

Theorem 5: $\mathcal{I}^{c}$ is continuous on $\mathbb{R}_{+}^{K}$. For an arbitrary sequence $\boldsymbol{p}^{(n)} \in \mathbb{R}_{+}^{K}$ with $\lim _{n \rightarrow \infty} \boldsymbol{p}^{(n)}=\boldsymbol{p}^{*}$ we have

$$
\lim _{n \rightarrow \infty} \mathcal{I}^{c}\left(\boldsymbol{p}^{(n)}\right)=\mathcal{I}^{c}\left(\boldsymbol{p}^{*}\right) .
$$

Proof: The proof is based on the results from Appendix I-B.

We need to show that $\mathcal{I}: \mathbb{R}_{+}^{K} \mapsto \mathbb{R}_{+}$is a continuous function, i.e., (25) holds for any sequence $\boldsymbol{p}^{(n)} \in \mathbb{R}_{+}^{K}$ with $\lim _{n \rightarrow \infty} \boldsymbol{p}^{(n)}=\boldsymbol{p}^{*}$.

Consider $\bar{\delta}^{(n)}$ and $\overline{\boldsymbol{p}}^{(n)}$, defined as in the proof of Lemma 8, with (94) and (95). Combining $\lim _{n \rightarrow \infty} \mathcal{I}\left(\overline{\boldsymbol{p}}^{(n)}\right)=\mathcal{I}\left(p^{*}\right)$ and (94) we have

$$
\limsup _{n \rightarrow \infty} \mathcal{I}\left(\boldsymbol{p}^{(n)}\right) \leq \mathcal{I}\left(\boldsymbol{p}^{*}\right)
$$

We introduce the vector $\underline{\boldsymbol{p}}^{(n)}$ with

$$
\left[\underline{\boldsymbol{p}}^{(n)}\right]_{k}=\underline{p}_{k}^{(n)}= \begin{cases}p_{k}^{(n)} & \text { if } p_{k}>0 \\ 0 & \text { if } p_{k}=0 .\end{cases}
$$

Since $\underline{\boldsymbol{p}}^{(n)} \leq \boldsymbol{p}^{(n)}$ we have

$$
\lim _{n \rightarrow \infty} \mathcal{I}\left(\underline{\boldsymbol{p}}^{(n)}\right) \leq \lim _{n \rightarrow \infty} \mathcal{I}\left(\boldsymbol{p}^{(n)}\right) .
$$

From Lemma 9 we know that the right-hand side limit of (28) exists, so

$$
\lim _{n \rightarrow \infty} \mathcal{I}\left(\underline{\boldsymbol{p}}^{(n)}\right)=\mathcal{I}\left(\boldsymbol{p}^{*}\right) .
$$

Combining (26), (28), and (29) we have

$$
\mathcal{I}\left(\boldsymbol{p}^{*}\right) \leq \liminf _{n \rightarrow \infty} \mathcal{I}\left(\boldsymbol{p}^{(n)}\right) \leq \limsup _{n \rightarrow \infty} \mathcal{I}\left(\boldsymbol{p}^{(n)}\right) \leq \mathcal{I}\left(\boldsymbol{p}^{*}\right) .
$$

Thus, (25) is fulfilled.

\section{The StRUCTURE OF INTERFERENCE FunCTIONS}

In this section we will study the structure of interference functions defined by A1, A2, A3. Other properties are optional. For example, it will be shown later in Section V that the result can be transferred to standard interference functions, by exploiting strict monotonicity A4.

In order to simplify the discussion, we will first confine the discussion to power vectors from $\mathbb{R}_{++}^{K}$. Later, we will use Theorem 4 to show that the results extend to $\mathbb{R}_{+}^{K}$.

We begin with some fundamental observations. Consider an arbitrary interference function $\mathcal{I}$ on $\mathbb{R}_{+}^{K}$, characterized by $\mathrm{A} 1$, A2, A3. Here, $K$ is an arbitrary finite dimension, so we can possibly include noise power as in Section II. From [22], Lemma 1 , we know that for $\boldsymbol{p}, \boldsymbol{q} \in \mathbb{R}_{++}^{K}$

$$
\begin{aligned}
& \mathcal{I}(\boldsymbol{p}) \leq \mathcal{I}(\boldsymbol{q}) \cdot \max _{k \in \mathcal{K}} \frac{p_{k}}{q_{k}} \\
& \mathcal{I}(\boldsymbol{p}) \geq \mathcal{I}(\boldsymbol{q}) \cdot \min _{k \in \mathcal{K}} \frac{p_{k}}{q_{k}} .
\end{aligned}
$$

These inequalities are fulfilled with equality if $\boldsymbol{p}=\boldsymbol{q}$. Thus

$$
\begin{aligned}
& \mathcal{I}(\boldsymbol{p})=\inf _{\boldsymbol{q}>0}\left(\mathcal{I}(\boldsymbol{q}) \max _{k \in \mathcal{K}} \frac{p_{k}}{q_{k}}\right)=\min _{\boldsymbol{q}>0}\left(\mathcal{I}(\boldsymbol{q}) \max _{k \in \mathcal{K}} \frac{p_{k}}{q_{k}}\right) \\
& \mathcal{I}(\boldsymbol{p})=\sup _{\boldsymbol{q}>0}\left(\mathcal{I}(\boldsymbol{q}) \min _{k \in \mathcal{K}} \frac{p_{k}}{q_{k}}\right)=\max _{\boldsymbol{q}>0}\left(\mathcal{I}(\boldsymbol{q}) \min _{k \in \mathcal{K}} \frac{p_{k}}{q_{k}}\right) .
\end{aligned}
$$

We can further exploit the following identities [24]:

$$
\begin{gathered}
\sup _{\boldsymbol{v}>0} \frac{\sum_{k \in \mathcal{K}} v_{k} p_{k}}{\sum_{k \in \mathcal{K}} v_{k} q_{k}}=\sup _{\boldsymbol{w}>0,\|\boldsymbol{w}\|_{1}=1} \frac{\prod_{k \in \mathcal{K}}\left(p_{k}\right)^{w_{k}}}{\prod_{k \in \mathcal{K}}\left(q_{k}\right)^{w_{k}}}=\max _{k \in \mathcal{K}} \frac{p_{k}}{q_{k}} \\
\inf _{\boldsymbol{v}>0} \frac{\sum_{k \in \mathcal{K}} v_{k} p_{k}}{\sum_{k \in \mathcal{K}} v_{k} q_{k}}=\inf _{\boldsymbol{w}>0,\|\boldsymbol{w}\|_{1}=1} \frac{\prod_{k \in \mathcal{K}}\left(p_{k}\right)^{w_{k}}}{\prod_{k \in \mathcal{K}}\left(q_{k}\right)^{w_{k}}}=\min _{k \in \mathcal{K}} \frac{p_{k}}{q_{k}} .
\end{gathered}
$$

For $\boldsymbol{w}, \boldsymbol{v}>0,\|\boldsymbol{w}\|_{1}=1$, we introduce functions

$$
\begin{aligned}
& G_{\mathcal{I}}(\boldsymbol{q}, \boldsymbol{p}, \boldsymbol{v}):=\left(\frac{\mathcal{I}(\boldsymbol{q})}{\sum_{l \in \mathcal{K}} v_{l} q_{l}}\right) \cdot \sum_{k \in \mathcal{K}} v_{k} p_{k} \\
& F_{\mathcal{I}}(\boldsymbol{q}, \boldsymbol{p}, \boldsymbol{w}):=\left(\frac{\mathcal{I}(\boldsymbol{q})}{\prod_{l \in \mathcal{K}}\left(q_{l}\right)^{w_{l}}}\right) \cdot \prod_{k \in \mathcal{K}}\left(p_{k}\right)^{w_{k}} .
\end{aligned}
$$

The next theorem is a direct consequence of (32) and (33).

Theorem 6: Consider an arbitrary interference function $\mathcal{I}$. For all $\boldsymbol{p}>0$ we have

$$
\begin{aligned}
\mathcal{I}(\boldsymbol{p}) & =\inf _{\boldsymbol{q}>0} \sup _{\boldsymbol{v}>0} G_{\mathcal{I}}(\boldsymbol{q}, \boldsymbol{p}, \boldsymbol{v})=\sup _{\boldsymbol{q}>0} \inf _{\boldsymbol{v}>0} G_{\mathcal{I}}(\boldsymbol{q}, \boldsymbol{p}, \boldsymbol{v}) \\
\mathcal{I}(\boldsymbol{p}) & =\inf _{\boldsymbol{q}>0} \sup _{\substack{\boldsymbol{w}>0 \\
\|\boldsymbol{w}\|_{1}=1}} F_{\mathcal{I}}(\boldsymbol{q}, \boldsymbol{p}, \boldsymbol{w}) \\
& =\sup _{\boldsymbol{q}>0} \inf _{\| \boldsymbol{w}>0}^{\boldsymbol{w} \|_{1}=1} F_{\mathcal{I}}(\boldsymbol{q}, \boldsymbol{p}, \boldsymbol{w}) .
\end{aligned}
$$

Theorem 6 shows that any interference function has a sup-inf and inf-sup characterization, involving functions $G_{\mathcal{I}}(\boldsymbol{q}, \boldsymbol{p}, \boldsymbol{v})$ and $F_{\mathcal{I}}(\boldsymbol{q}, \boldsymbol{p}, \boldsymbol{w})$. These functions fulfill the axioms A1, A2, A3 (with respect to the variable $\boldsymbol{p}$ ), so they can be regarded as elementary interference functions. This result stands in an interesting relationship with recent results [22], where different max-min and min-max representations were shown.

Note, that (38) and (39) are not saddle point characterizations, because we do not only interchange the optimization order, but also the domain. Representation (38) will be used in the following Sections IV-A and IV-B, where we analyze convex and concave interference functions. Representation (39) will be needed later in Sections IV-C, where log-convex interference functions will be analyzed. 
We begin by focusing on (38). By exchanging inf and sup, we obtain for all $\boldsymbol{p}>0$

$$
\begin{aligned}
& \mathcal{I}(\boldsymbol{p}) \geq \sup _{\boldsymbol{v}>0} \inf _{\boldsymbol{q}>0} G_{\mathcal{I}}(\boldsymbol{q}, \boldsymbol{p}, \boldsymbol{v})=\underline{\mathcal{I}}(\boldsymbol{p}) \\
& \mathcal{I}(\boldsymbol{p}) \leq \inf _{\boldsymbol{v}>0} \sup _{\boldsymbol{q}>0} G_{\mathcal{I}}(\boldsymbol{q}, \boldsymbol{p}, \boldsymbol{v})=\overline{\mathcal{I}}(\boldsymbol{p}) .
\end{aligned}
$$

The resulting functions $\underline{\mathcal{I}}(\boldsymbol{p})$ and $\overline{\mathcal{I}}(\boldsymbol{p})$ are minorants and majorants, respectively, according to the following definition.

Definition 6: An interference function $\underline{\mathcal{I}}(\boldsymbol{p})$ is said to be a minorant of $\mathcal{I}(\boldsymbol{p})$ if $\mathcal{I}(\boldsymbol{p}) \leq \mathcal{I}(\boldsymbol{p})$ for all $\boldsymbol{p} \in \mathcal{P}$, where $\mathcal{P}$ is the domain of $\mathcal{I}$. An interference function $\overline{\mathcal{I}}(\boldsymbol{p})$ is said to be a majorant if $\overline{\mathcal{I}}(\boldsymbol{p}) \geq \mathcal{I}(\boldsymbol{p})$ for all $\boldsymbol{p} \in \mathcal{P}$.

The minorant (40) and the majorant (41) will play an important role for the analysis of convex and concave interference functions, respectively. This will be shown in the next Sections IV-A and IV-B.

\section{A. Convex Interference Functions}

The following lemma was derived in [15].

Lemma 2: An interference function $\mathcal{I}(\boldsymbol{p})$ is convex if and only if there exists a nonempty bounded downward-comprehensive closed convex set $\mathcal{V}_{\mathcal{I}} \subset \mathbb{R}_{+}^{K}$ such that

$$
\mathcal{I}(\boldsymbol{p})=\max _{\boldsymbol{v} \in \mathcal{V}_{\mathcal{I}}} \sum_{k \in \mathcal{K}} v_{k} p_{k}, \quad \text { for all } \boldsymbol{p}>0 .
$$

The corresponding version for concave interference functions was already discussed in Section III-B. Here, similar conclusions can be drawn from Lemma 2 . That is, the power minimization problem (10) can be solved with superlinear convergence, by exploiting that any convex interference function can be expressed as a maximum of linear functions.

In this section, we will derive an alternative way of expressing a convex interference function as a maximum of linear functions. This new representation differs from (42) in that the optimization is not over a specific coefficient set. The behavior of $\mathcal{I}$ is instead captured by a function $\underline{g}_{\mathcal{I}}$ defined as follows.

$$
\begin{aligned}
\underline{g}_{\mathcal{I}}(\boldsymbol{v}) & =\inf _{\boldsymbol{q}>0}\left(\frac{\mathcal{I}(\boldsymbol{q})}{\sum_{l \in \mathcal{K}} v_{l} q_{l}}\right), \quad \boldsymbol{v} \supsetneqq \mathbf{0} \\
& =\inf _{\substack{\boldsymbol{q}>0 \\
\|\boldsymbol{q}\|_{1}=1}}\left(\frac{\mathcal{I}(\boldsymbol{q})}{\sum_{l \in \mathcal{K}} v_{l} q_{l}}\right) .
\end{aligned}
$$

We begin by showing that the infimum (43) is attained.

Lemma 3: For any $\boldsymbol{v}>0$, there is a $\hat{\boldsymbol{q}}=\hat{\boldsymbol{q}}(\boldsymbol{v}) \geq 0$, with $\|\hat{\boldsymbol{q}}\|_{1}=1$, such that

$$
\underline{g}_{\mathcal{I}}(\boldsymbol{v})=\frac{\mathcal{I}(\hat{\boldsymbol{q}})}{\sum_{l \in \mathcal{K}} v_{l} \hat{q}_{l}}=\min _{\substack{\boldsymbol{q} \geq 0 \\\|\boldsymbol{q}\|_{1}=1}}\left(\frac{\mathcal{I}(\boldsymbol{q})}{\sum_{l \in \mathcal{K}} v_{l} q_{l}}\right) .
$$

Proof: Since $\boldsymbol{v}>0$, we have $\sum_{l \in \mathcal{K}} v_{l} q_{l}>0$ for all $\boldsymbol{q} \geq 0$. So we can take the infimum (43) over the compact domain $\{\boldsymbol{q} \geq$ $\left.0:\|\boldsymbol{q}\|_{1}=1\right\}$. The inverse of a continous positive function is continuous. Also, $\mathcal{I}(\boldsymbol{p})$ is continuous on $\mathbb{R}_{++}^{K}$ [14]. Theorem 5 shows that $\mathcal{I}(\boldsymbol{p})$ has a unique continuation on the boundary, so continuity extends to $\mathbb{R}_{+}^{K}$. Any continuous real-valued function attains a minimum over a compact set, thus (44) holds.

Consider the minorant (40). With (43), we can rewrite (40) as

$$
\begin{aligned}
\underline{I}(\boldsymbol{p}) & =\sup _{\boldsymbol{v}>0}\left(\inf _{\boldsymbol{q}>0}\left(\frac{\mathcal{I}(\boldsymbol{q})}{\sum_{l \in \mathcal{K}} v_{l} q_{l}}\right) \sum_{k \in \mathcal{K}} v_{k} p_{k}\right) \\
& =\sup _{\boldsymbol{v}>0}\left(\underline{g}_{\mathcal{I}}(\boldsymbol{v}) \sum_{k \in \mathcal{K}} v_{k} p_{k}\right) .
\end{aligned}
$$

Any pointwise supremum of linear functions is convex, so the minorant $\mathcal{I}(\boldsymbol{p})$ is a convex interference function.

The next theorem and the following corollary show that the convex minorant $\underline{\mathcal{I}}(\boldsymbol{p})$ is best possible, and any convex interference function has a saddle-point characterization.

Theorem 7: $\mathcal{I}$ is a convex interference function if and only if $\underline{I}(\boldsymbol{p})=\mathcal{I}(\boldsymbol{p})$ for all $\boldsymbol{p}>0$, i.e.,

$$
\mathcal{I}(\boldsymbol{p})=\inf _{\boldsymbol{q}>0} \sup _{\boldsymbol{v}>0} G_{\mathcal{I}}(\boldsymbol{q}, \boldsymbol{p}, \boldsymbol{v})=\sup _{\boldsymbol{v}>0} \inf _{\boldsymbol{q}>0} G_{\mathcal{I}}(\boldsymbol{q}, \boldsymbol{p}, \boldsymbol{v}) .
$$

Proof: see Appendix I-C.

Corollary 2: Let $\mathcal{I}$ be an arbitrary interference function, then $\underline{I}(\boldsymbol{p})$ is the greatest convex minorant of $\mathcal{I}(\boldsymbol{p})$.

Proof: see Appendix I-D.

Note, that the greatest convex minorant was also studied in [15]. However, [15] uses a different approach, based on the representation (42). The alternative representation provided here helps to better understand the structure of such functions. The convex minorant (resp. concave majorant) is also interesting from a practical point of view, because convex/concave interference functions typically lead to efficient algorithmic solutions (see, e.g., [17]). These results provide best-possible convex/concave approximations for any (possibly nonconvex) interference function.

Next, we show that the inverse

$$
\mathcal{I}_{1}(\boldsymbol{v})=\frac{1}{\underline{g}_{\mathcal{I}}(\boldsymbol{v})}
$$

is an interference function. Note, that $\mathcal{I}_{1}(\boldsymbol{v})$ is always defined because of the following property: If there exists a $\boldsymbol{v}^{\prime}>0$ such that $\underline{g}_{\mathcal{I}}\left(\boldsymbol{v}^{\prime}\right)>0$, then $\underline{g}_{\mathcal{T}}(\boldsymbol{v})>0$ for all $\boldsymbol{v}>0$.

For arbitrary $\boldsymbol{v}>0$ and $\lambda>0$ we have

$$
\underline{g}_{\mathcal{I}}(\lambda \boldsymbol{v})=\frac{1}{\lambda} \cdot \underline{g}_{\mathcal{I}}(\boldsymbol{v}) \text {. }
$$

If $\boldsymbol{v}^{(1)} \geq \boldsymbol{v}^{(2)}$, then $\underline{g}_{\mathcal{I}}\left(\boldsymbol{v}^{(1)}\right) \leq \underline{g}_{\mathcal{I}}\left(\boldsymbol{v}^{(2)}\right)$, so $\mathcal{I}_{1}(\boldsymbol{v})$ fulfills axioms A2, A3.

Now, we show positivity (A1). For any $\boldsymbol{v}>0$, with $\|\boldsymbol{v}\|_{1}=1$, Lemma 3 implies

$$
\underline{g}_{\mathcal{I}}(\boldsymbol{v})=\min _{\substack{\boldsymbol{q} \geq 0 \\\|\boldsymbol{q}\|_{1}=1}}\left(\frac{\mathcal{I}(\boldsymbol{q})}{\sum_{l \in \mathcal{K}} v_{l} q_{l}}\right) \leq \frac{\frac{1}{K} \mathcal{I}(\mathbf{1})}{\frac{1}{K} \sum_{l \in \mathcal{K}} v_{l}}=\mathcal{I}(\mathbf{1}) .
$$

That is, $\underline{g}_{\mathcal{I}}(\boldsymbol{v})$ is bounded from above by some constant $\mathcal{I}(\mathbf{1})$, and we have

$$
\mathcal{I}_{1}(\boldsymbol{v})=\frac{1}{\underline{g}_{\mathcal{I}}(\boldsymbol{v})} \geq \frac{1}{\mathcal{I}(\mathbf{1})}>0
$$


Thus, $\mathcal{I}_{1}$ is lower bounded for any $\boldsymbol{v}$. With Theorem 4 we can define $\mathcal{I}_{1}$ for $\boldsymbol{v} \geq 0$, with $\|\boldsymbol{v}\|_{1}=1$. Then, $\underline{g}_{\mathcal{I}}(\boldsymbol{v})=1 / \mathcal{I}_{1}(\boldsymbol{v})$ is continuous on $\left\{\boldsymbol{v} \geq 0:\|\boldsymbol{v}\|_{1}=1\right\}$.

Thus, the function $\mathcal{I}_{1}(\boldsymbol{v})=1 / \underline{g}_{\mathcal{I}}(\boldsymbol{v})$ fulfills the axioms A1, A2, A3. We also have

$$
\mathcal{I}_{1}(\boldsymbol{v})=\frac{1}{\inf \underset{\|\boldsymbol{q}\|_{1}=1}{\boldsymbol{q}>0}\left(\frac{\mathcal{I}(\boldsymbol{q})}{\sum_{l \in \mathcal{K}} v_{l} q_{l}}\right)}=\sup _{\substack{\boldsymbol{q}>0 \\\|\boldsymbol{q}\|_{1}=1}} \frac{\sum_{l \in \mathcal{K}} v_{l} q_{l}}{\mathcal{I}(\boldsymbol{q})} .
$$

The pointwise supremum of linear functions is convex. Hence, $\mathcal{I}_{1}(\boldsymbol{v})$ is a convex interference function.

Theorem 8: Let $\mathcal{I}$ be an arbitrary interference function, then $\underline{g}_{\mathcal{T}}(\boldsymbol{v})$ is continuous on $\mathbb{R}_{+}^{K}$, and there exists a nonempty bounded downward-comprehensive closed convex set $\underline{\mathcal{W}} \subset \mathbb{R}_{+}^{K}$ such that

$$
\underline{g}_{\mathcal{I}}(\boldsymbol{v})=\frac{1}{\max _{\boldsymbol{w} \in \underline{\mathcal{W}}} \sum_{k \in \mathcal{K}} w_{k} v_{k}}=\min _{\boldsymbol{w} \in \underline{\mathcal{W}}} \frac{1}{\sum_{k \in \mathcal{K}} w_{k} v_{k}} .
$$

Proof: Since $\mathcal{I}_{1}(\boldsymbol{v})$ is a convex interference function, we know from Lemma 2 that (49) holds for any $\boldsymbol{v} \in \mathbb{R}_{++}^{K}$. The denominator in (49) is strictly positive, so $\underline{g}_{\mathcal{T}}$ is continuous as the pointwise minimum of continuous functions. From Theorem 5 we know that continuity extends to $\mathbb{R}_{+}^{K}$.

With the continuity shown by Theorem 8 and property (45), we know that the minorant $\underline{\mathcal{I}}$ can be rewritten as

$$
\underline{\mathcal{I}}(\boldsymbol{p})=\max _{\substack{\boldsymbol{v} \geq 0 \\\|\boldsymbol{v}\|_{1}=1}} \underline{g}_{\mathcal{I}}(\boldsymbol{v}) \cdot \sum_{k \in \mathcal{K}} v_{k} p_{k}, \quad \boldsymbol{p}>0 .
$$

That is, the supremum can be replaced by a maximum over a compact set, and $\underline{g}_{\mathcal{I}}$ is defined as in (43). With Theorem 7 and (50), the following result is shown.

Theorem 9: $\mathcal{I}$ is a convex interference function if and only if

$$
\mathcal{I}(\boldsymbol{p})=\max _{\substack{\boldsymbol{v} \geq 0 \\\|\boldsymbol{v}\|_{1}=1}} \underline{g}_{\mathcal{I}}(\boldsymbol{v}) \cdot \sum_{k \in \mathcal{K}} v_{k} p_{k} \quad \text { for all } \boldsymbol{p}>0 .
$$

Comparing (51) with (42), we observe two different ways of expressing a convex interference function as the maximum of linear functions. In (42), the coefficient set $\mathcal{V}_{\mathcal{I}}$ is used to incorporate the properties of $\mathcal{I}$ (see [15] for details), while (51) uses the function $\underline{g}_{\mathcal{I}}$.

\section{B. Concave Interference Functions}

Next, similar results are shown for concave interference functions. Such functions result, for example, from adaptive receive strategies, like the beamforming interference discussed in Appendix I-A.

First, recall Lemma 1, which shows that any concave interference function can be expressed as the minimum over affine functions, where the optimization is over a closed comprehensive convex coefficient set.
We will now derive a different characterization based on the function

$$
\bar{g}_{\mathcal{I}}(\boldsymbol{v})=\sup _{\substack{\boldsymbol{q}>0 \\\|\boldsymbol{q}\|_{1}=1}}\left(\frac{\mathcal{I}(\boldsymbol{q})}{\sum_{l \in \mathcal{K}} v_{l} q_{l}}\right), \quad \boldsymbol{v} \supsetneqq \mathbf{o} .
$$

The supremum (52) is always attained, as shown by the next lemma.

Lemma 4: For any $\boldsymbol{v}>0$, there is a $\hat{\boldsymbol{q}}=\hat{\boldsymbol{q}}(\boldsymbol{v}) \geq 0$, with $\|\hat{\boldsymbol{q}}\|_{1}=1$, such that

$$
\bar{g}_{\mathcal{I}}(\boldsymbol{v})=\frac{\mathcal{I}(\hat{\boldsymbol{q}})}{\sum_{l \in \mathcal{K}} v_{l} \hat{q}_{l}}=\max _{\substack{\boldsymbol{q} \geq 0 \\\|\boldsymbol{q}\|_{1}=1}}\left(\frac{\mathcal{I}(\boldsymbol{q})}{\sum_{l \in \mathcal{K}} v_{l} q_{l}}\right) .
$$

The proof is similar to the proof of Lemma 3.

With (52) we can rewrite the majorant (41) as

$$
\begin{aligned}
\overline{\mathcal{I}}(\boldsymbol{p}) & =\inf _{\boldsymbol{v}>0} \sup _{\boldsymbol{q}>0}\left(\frac{\mathcal{I}(\boldsymbol{q})}{\sum_{l \in \mathcal{K}} v_{l} q_{l}}\right) \sum_{k \in \mathcal{K}} v_{k} p_{k} \\
& =\inf _{\boldsymbol{v}>0} \bar{g}_{\mathcal{I}}(\boldsymbol{v}) \sum_{k \in \mathcal{K}} v_{k} p_{k} .
\end{aligned}
$$

The infimum of linear functions is concave, so $\overline{\mathcal{I}}(\boldsymbol{p})$ is a concave interference function. The next theorem and the following corollary show that the concave majorant $\overline{\mathcal{I}}(\boldsymbol{p})$ is best possible, and any concave interference function has a saddle-point characterization.

Theorem 10: $\mathcal{I}$ is a concave interference function if and only if $\overline{\mathcal{I}}(\boldsymbol{p})=\mathcal{I}(\boldsymbol{p})$ for all $\boldsymbol{p}>0$, i.e.,

$$
\mathcal{I}(\boldsymbol{p})=\sup _{\boldsymbol{q}>0} \inf _{\boldsymbol{v}>0} G_{\mathcal{I}}(\boldsymbol{q}, \boldsymbol{p}, \boldsymbol{v})=\inf _{\boldsymbol{v}>0} \sup _{\boldsymbol{q}>0} G_{\mathcal{I}}(\boldsymbol{q}, \boldsymbol{p}, \boldsymbol{v}) .
$$

The proof is similar to the proof of Theorem 7 in Appendix I-C.

Corollary 3: Let $\mathcal{I}$ be an arbitrary interference function, then $\overline{\mathcal{I}}(\boldsymbol{p})$ is the least concave majorant of $\mathcal{I}(\boldsymbol{p})$.

Corollary 3 stands in interesting relation to the analysis of the least concave majorant in [15], similar to the convex case. The proof is similar to the proof of Corollary 2 .

Next, consider the function

$$
\mathcal{I}_{2}(\boldsymbol{v})=\frac{1}{\bar{g}_{\mathcal{I}}(\boldsymbol{v})}=\inf _{\substack{\boldsymbol{q}>0 \\\|\boldsymbol{q}\|_{1}=1}} \frac{\sum_{l \in \mathcal{K}} v_{l} q_{l}}{\mathcal{I}(\boldsymbol{q})} .
$$

Similar to the convex case, it can be shown that $\mathcal{I}_{2}(\boldsymbol{v})$ is a concave interference function. This is used for proving the following result. The proof is similar to the proof of Theorem 8 .

Theorem 11: Let $\mathcal{I}$ be an arbitrary interference function, then $\bar{g}_{\mathcal{I}}(\boldsymbol{v})$ is continuous on $\mathbb{R}_{+}^{K}$, and there exists a nonempty upward-comprehensive closed convex set $\overline{\mathcal{W}} \subset \mathbb{R}_{+}^{K}$ such that

$$
\bar{g}_{\mathcal{I}}(\boldsymbol{v})=\frac{1}{\min _{\boldsymbol{w} \in \overline{\mathcal{W}}} \sum_{k \in \mathcal{K}} w_{k} v_{k}}=\max _{\boldsymbol{w} \in \overline{\mathcal{W}}} \frac{1}{\sum_{k \in \mathcal{K}} w_{k} v_{k}} .
$$


With the continuity shown by Theorem 11 and (54) we know that the majorant $\overline{\mathcal{I}}$ can be rewritten as

$$
\overline{\mathcal{I}}(\boldsymbol{p})=\min _{\substack{\boldsymbol{v} \geq 0 \\\|\boldsymbol{v}\|_{1}=1}} \bar{g}_{\mathcal{I}}(\boldsymbol{v}) \cdot \sum_{k \in \mathcal{K}} v_{k} p_{k}, \quad \boldsymbol{p}>0
$$

With (58) and Theorem 10 we obtain the following result.

Theorem 12: $\mathcal{I}$ is a concave interference function if and only if

$$
\mathcal{I}(\boldsymbol{p})=\min _{\substack{\boldsymbol{v} \geq 0 \\\|\boldsymbol{v}\|_{1}=1}} \bar{g}_{\mathcal{I}}(\boldsymbol{v}) \cdot \sum_{k \in \mathcal{K}} v_{k} p_{k} \quad \text { for all } \boldsymbol{p}>0
$$

Comparing (59) with (15) in Lemma 1, we observe two different ways of expressing a concave interference function as the minimum of linear functions. In (15), the coefficient set $\mathcal{V}_{\mathcal{I}}$ is used to incorporate the properties of $\mathcal{I}$ (see [15] for details), while (59) uses the function $\bar{g}_{\mathcal{I}}$.

\section{Log-Convex Interference Functions}

Logarithmically convex (log-convex) interference functions (see Definition 2) are an interesting generalization of convex interference functions, which in turn include the often-used linear interference functions. Many well-known concepts from power control, like the Perron-Frobenius theory of nonnegative matrices, have counterparts in the framework of log-convex interference functions [16].

In this section we analyze the structure of log-convex interference functions on the basis of the sup-inf characterization (39). This approach complements the results [16], where a different approach was chosen.

We begin by introducing the function

$$
\underline{f}_{\mathcal{I}}(\boldsymbol{w})=\inf _{\substack{\boldsymbol{q}>0 \\\|\boldsymbol{q}\|_{1}=1}} \frac{\mathcal{I}(\boldsymbol{q})}{\prod_{l \in \mathcal{K}}\left(q_{l}\right)^{w_{l}}}, \quad \boldsymbol{w} \supsetneqq \mathbf{0} .
$$

The function $f_{\mathcal{T}}$ plays a similar role for log-convex interference functions as did $\underline{g}_{\mathcal{I}}$ for the convex case. It was observed in [16] that $\underline{f}_{\mathcal{T}}(\boldsymbol{w})$ is log-concave on $\mathbb{R}_{+}^{K}$. However it is not an interference function.

By exchanging inf and sup in (39) we obtain for all $\boldsymbol{p}>0$

$$
\begin{aligned}
\mathcal{I}(\boldsymbol{p}) & \geq \sup _{\substack{\boldsymbol{w}>0 \\
\|\boldsymbol{w}\|_{1}=1}} \inf _{\boldsymbol{q}>0} F_{\mathcal{I}}(\boldsymbol{q}, \boldsymbol{p}, \boldsymbol{w}) \\
& =\sup _{\substack{\boldsymbol{w}>0 \\
\|\boldsymbol{w}\|_{1}=1}} \underline{f}_{\mathcal{I}}(\boldsymbol{w}) \prod_{k \in \mathcal{K}}\left(p_{k}\right)^{w_{k}}=: \underline{\mathcal{I}}_{2}(\boldsymbol{p}) .
\end{aligned}
$$

The function $\mathcal{I}_{2}$ is a minorant of $\mathcal{I}$. It was shown in [16] that the infimum (61) is attained.

$$
\underline{\mathcal{I}}_{2}(\boldsymbol{p})=\max _{\boldsymbol{w} \geq 0} \underline{f}_{\mathcal{I}}(\boldsymbol{w}) \prod_{k \in \mathcal{K}}\left(p_{k}\right)^{w_{k}} .
$$

Note, that this property is enabled by the assumed log-convexity of $\mathcal{I}$. For general interference functions it is not clear whether the supremum is attained.
Theorem 13: $\mathcal{I}$ is a log-convex interference function if and only if $\underline{\underline{I}}_{2}(\boldsymbol{p})=\mathcal{I}(\boldsymbol{p})$, i.e.,

$$
\begin{aligned}
\mathcal{I}(\boldsymbol{p}) & =\inf _{\boldsymbol{q}>0} \sup _{\substack{\boldsymbol{w}>0 \\
\|\boldsymbol{w}\|_{1}=1}} F_{\mathcal{I}}(\boldsymbol{q}, \boldsymbol{p}, \boldsymbol{w}) \\
& =\sup _{\substack{\boldsymbol{w}>0 \\
\|\|_{1}=1}} \inf _{\boldsymbol{q}>0} F_{\mathcal{I}}(\boldsymbol{q}, \boldsymbol{p}, \boldsymbol{w}) .
\end{aligned}
$$

Proof: This follows from the results [16]. An alternative proof is based on the max-min characterization (39), similar to the proof of Theorem 7 .

Corollary 4: Let $\mathcal{I}$ be an arbitrary interference function, then $\underline{I}_{2}$ is the greatest log-convex minorant.

Proof: This also follows from the results [16]. Alternatively, it can be shown in a similar way as Corollary 2 .

\section{APPLiCATION to StANDARD INTERFERENCE FUNCTIONS}

We now show how the structure results from Section IV can be applied to standard interference functions as defined in Section III-A. In particular, we show that convexity and log-convexity, which were studied within the framework A1, $\mathrm{A} 2, \mathrm{~A} 3$ in [15], [16], and [22], is preserved when switching to the framework of standard interference functions.

As in the previous section we focus on strictly positive power vectors from $\mathbb{R}_{++}^{K_{u}+1}$. This is a technical restriction compared to the previously used $\mathcal{P}$, as defined in (18), where transmission powers are allowed to be zero. However, we can extend the following results to sets $\underline{\mathcal{P}}$ by using the continuity result found in Section III-D.

\section{A. Characterization of Weakly Standard Interference Functions}

We now use Theorem 3 in order to transfer the structure results from Section IV to weakly standard interference functions. To this end, consider the function $\underline{g}_{\mathcal{I}}$ as defined by (43). Assume that a weakly standard interference function $Y$ is given. With (19) we obtain an interference function $\mathcal{I}=\mathcal{I}_{Y}$ with dimension $K=K_{u}+1$, where the last component stands for constant noise. We have

$$
\begin{aligned}
\underline{g}_{\mathcal{I}}(\boldsymbol{v}) & =\inf _{\boldsymbol{q} \in \mathbb{R}_{++}^{K_{u}+1}} \frac{\underline{q}_{K_{u}+1} \cdot Y\left(\frac{q_{1}}{\underline{q}_{K_{u}+1}}, \ldots, \frac{q_{K_{u}}}{\underline{q}_{K_{u}+1}}\right)}{\sum_{l=1}^{K_{u}+1} v_{l} q_{l}} \\
& =\inf _{\boldsymbol{q} \in \mathbb{R}_{++}^{K_{u}+1}} \frac{Y\left(\frac{q_{1}}{\underline{q}_{K_{u}+1}}, \ldots, \frac{q_{K_{u}}}{\underline{q}_{K_{u}+1}}\right)}{\sum_{l=1}^{K_{u}} v_{l} \frac{q_{l}}{\underline{q}_{K_{u}+1}}+v_{K_{u}+1}} \\
& =\inf _{\tilde{\boldsymbol{q}} \in \mathbb{R}_{++}^{K_{u}}} \frac{Y\left(\tilde{q}_{1}, \ldots, \tilde{q}_{K_{u}}\right)}{\sum_{l=1}^{K_{u}} v_{l} \tilde{q}_{K_{u}+1}+v_{K_{u}+1}}=: \underline{g}_{Y}(\boldsymbol{v}) .
\end{aligned}
$$

Using the structure results from Section IV, we can provide necessary and sufficient conditions for the convexity of standard interference functions: 
Theorem 14: A weakly standard interference function $Y$ is convex on $\mathbb{R}_{+}^{K_{u}}$ if and only if one of the following equivalent statements hold.

- The interference function $\mathcal{I}_{Y}$, as defined by (19), is convex.

- There exists a nonempty bounded downward-comprehensive closed convex set $\mathcal{V} \subset \mathbb{R}_{+}^{K_{u}+1}$ such that for all $\boldsymbol{p}>0$

$$
Y(\boldsymbol{p})=\max _{\boldsymbol{v} \in \mathcal{V}}\left(\sum_{k \in \mathcal{K}_{u}} v_{k} p_{k}+v_{K_{u}+1}\right) .
$$

- There is a function $\underline{g}_{Y}(\boldsymbol{v})$, as defined by (63), such that

$$
Y(\boldsymbol{p})=\max _{\substack{\boldsymbol{v}>0 \\\|\boldsymbol{v}\|_{1}=1}} \underline{g}_{Y}(\boldsymbol{v})\left(\sum_{k \in \mathcal{K}_{u}} v_{k} p_{k}+v_{K_{u}+1}\right)
$$

Proof: We have $Y(\boldsymbol{p})=\mathcal{I}_{Y}(\boldsymbol{p}, 1)$, so the second statement follows directly from Theorem 2 , and the last statement follows from Theorem 9.

It remains to prove the first statement. If $\mathcal{I}_{Y}$ is convex then $Y(\boldsymbol{p})=\mathcal{I}_{Y}(\boldsymbol{p}, 1)$ is convex on $\mathbb{R}_{+}^{K_{u}}$, since one coordinate is constant. Conversely, we need to show that any convex weakly standard interference function leads to a convex interference function $\mathcal{I}_{Y}$, as defined by (19). To this end, we introduce the conjugate function

$$
\bar{Y}^{*}(\boldsymbol{v})=\sup _{\boldsymbol{p} \in \mathbb{R}_{++}^{K_{u}}}\left(\sum_{l=1}^{K_{u}} v_{l} p_{l}-Y(\boldsymbol{p})\right) .
$$

Corollary 1 states that $Y$ is continuous on $\mathbb{R}_{++}^{K_{u}}$ and the results of Section III-D show that it has a unique continuation on the boundary. The conjugate $\bar{Y}^{*}(\boldsymbol{v})$ is lower semi-continuous as the pointwise supremum of continuous functions. It is also convex on the domain

$$
\mathcal{V}_{0}=\left\{\boldsymbol{v} \in \mathbb{R}^{K_{u}}: \bar{Y}^{*}(\boldsymbol{v})<+\infty\right\}
$$

For arbitrary $v \in \mathcal{V}_{0}$ and $\lambda>1$ we have

$$
\begin{aligned}
\bar{Y}^{*}(\boldsymbol{v}) & =\sup _{\boldsymbol{p} \in \mathbb{R}_{++}^{K_{u}}}\left(\lambda \sum_{l=1}^{K_{u}} v_{l} p_{l}-Y(\lambda \boldsymbol{p})\right) \\
& \geq \sup _{\boldsymbol{p} \in \mathbb{R}_{++}^{K_{u}}}\left(\lambda \sum_{l=1}^{K_{u}} v_{l} p_{l}-\lambda Y(\boldsymbol{p})\right)=\lambda \bar{Y}^{*}(\boldsymbol{v}) .
\end{aligned}
$$

Thus $(\lambda-1) \bar{Y}^{*}(\boldsymbol{v}) \leq 0$. This can only be fulfilled if $\bar{Y}^{*}(\boldsymbol{v}) \leq 0$. So, $\mathcal{V}_{0}$ can be expressed as the set of all $\boldsymbol{v} \in \mathbb{R}^{K_{u}}$ such that $\bar{Y}^{*}(\boldsymbol{v}) \leq 0$. Using similar arguments as in [15], it can also be shown that $\boldsymbol{v} \in \mathcal{V}_{0}$ implies $\boldsymbol{v} \geq 0$.

Since $Y$ is convex and continuous, the conjugate of the conjugate (the biconjugate) of $Y$ is $Y$ again, i.e.,

$$
\begin{aligned}
Y(\boldsymbol{p}) & =\sup _{\boldsymbol{v} \in \mathcal{V}_{0}}\left(\sum_{l=1}^{K_{u}} v_{l} p_{l}-\bar{Y}^{*}(\boldsymbol{v})\right) \\
& =\sup _{\boldsymbol{v}>0: \bar{Y}^{*}(\boldsymbol{v}) \leq 0}\left(\sum_{l=1}^{K_{u}} v_{l} p_{l}-\bar{Y}^{*}(\boldsymbol{v})\right) .
\end{aligned}
$$

With (69) and (19) we have

$$
\begin{aligned}
\mathcal{I}_{Y}(\underline{\boldsymbol{p}}) & =\underline{p}_{K_{u}+1} \sup _{\boldsymbol{v}>0: \bar{Y}^{*}(\boldsymbol{v}) \leq 0}\left(\sum_{l=1}^{K_{u}} v_{l} \frac{p_{\boldsymbol{l}}}{\underline{p}_{K_{u}+1}}-\bar{Y}^{*}(\boldsymbol{v})\right) \\
& =\sup _{\boldsymbol{v}>0: \bar{Y}^{*}(\boldsymbol{v}) \leq 0}\left(\sum_{l=1}^{K_{u}} v_{l} p_{\boldsymbol{l}}-\underline{p}_{K_{u}+1} \bar{Y}^{*}(\boldsymbol{v})\right) .
\end{aligned}
$$

The supremum of linear functions is convex, thus $\mathcal{I}$ is a convex interference function.

The property $\bar{Y}^{*}(\boldsymbol{v}) \leq 0$ is important since otherwise monotonicity would not be fulfilled and $\mathcal{I}$ would not be an interference function. Showing this property is actually not required for the proof because Theorem 3 already states that $\mathcal{I}$ is an interference function. However, the proof shows this result directly. It thereby provides a better understanding of the fundamental structure of interference functions. For a more detailed analysis of convex and concave interference functions, the reader is referred to [15].

For concave interference functions similar results can be derived. To this end we introduce

$$
\begin{aligned}
\bar{g}_{\mathcal{I}}(\boldsymbol{v}) & =\sup _{\boldsymbol{q} \in \mathbb{R}_{++}^{K_{u}+1}} \frac{\underline{q}_{K_{u}+1} \cdot Y\left(\frac{q_{1}}{\underline{q}_{K_{u}+1}}, \ldots, \frac{q_{K_{u}}}{\underline{q}_{K_{u}+1}}\right)}{\sum_{\boldsymbol{l}=1}^{K_{u}+1} v_{l} q_{l}} \\
& =\sup _{\tilde{\boldsymbol{q}} \in \mathbb{R}_{++}^{K_{u}}} \frac{Y\left(\tilde{q}_{1}, \ldots, \tilde{q}_{K_{u}}\right)}{\sum_{l=1}^{K_{u}} v_{l} \tilde{q}_{K_{u}+1}+v_{K_{u}+1}}=: \bar{g}_{Y}(\boldsymbol{v})
\end{aligned}
$$

Theorem 15: A weakly standard interference function $Y$ is concave on $\mathbb{R}_{+}^{K_{u}}$ if and only if the following equivalent statements hold.

- The interference function $\mathcal{I}_{Y}$, as defined by (19), is concave.

- There exists a nonempty closed upward-comprehensive convex set $\mathcal{V} \subset \mathbb{R}_{+}^{K_{u}+1}$ such that for all $\boldsymbol{p}>0$

$$
Y(\boldsymbol{p})=\min _{\boldsymbol{v} \in \mathcal{V}}\left(\sum_{k \in \mathcal{K}_{u}} v_{k} p_{k}+v_{K_{u}+1}\right) \text {. }
$$

- There is a function $\bar{g}_{Y}(\boldsymbol{v})$, as defined by (71), such that

$$
Y(\boldsymbol{p})=\inf _{\substack{\boldsymbol{v}>0 \\\|\boldsymbol{v}\|_{1}=1}} \bar{g}_{Y}(\boldsymbol{v})\left(\sum_{k \in \mathcal{K}_{u}} v_{k} p_{k}+v_{K_{u}+1}\right) \text {. }
$$

Proof: The proof is similar to the proof of Theorem 14.

Similar to the functions $\mathcal{I}_{1}$ and $\mathcal{I}_{2}$ discussed in Section IV, we can show that the functions $1 / \bar{g}_{Y}(\boldsymbol{v})$ and $1 / \underline{g}_{Y}(\boldsymbol{v})$ are weakly standard interference functions. This implies that they are continuous on $\mathbb{R}_{++}^{K_{u}}$.

Next, we consider the log-convex case. As in Section II-A, $\log$-convexity is defined with respect to the variable $\boldsymbol{s}=\log \boldsymbol{p}$ (component-wise). The second part of the following theorem builds on previous results [16], where a function

$$
f_{\mathcal{I}}(\boldsymbol{w})=\inf _{\boldsymbol{p}>0} \frac{\mathcal{I}(\boldsymbol{p})}{\prod_{l \in \mathcal{K}}\left(p_{l}\right)^{w_{l}}}, \quad \boldsymbol{w} \in \mathbb{R}_{+}^{K_{u}}
$$


was used for analyzing the structure of of log-convex interference functions. The function $f_{\mathcal{I}}(\boldsymbol{w})$ is the conjugate of $\log \mathcal{I}(\exp s)[16]$.

Theorem 16: Let $Y$ be a weakly standard interference function and $\mathcal{I}_{Y}$ be defined by (19). Then $Y(\exp s)$ is log-convex if and only if $\mathcal{I}_{Y}(\exp \underline{s})$ is log-convex. In this case, we have

$$
Y(\boldsymbol{p})=\sup _{\boldsymbol{w}: f_{\mathcal{I}}(\boldsymbol{w})>0} f_{\mathcal{I}}(\boldsymbol{w}) \prod_{l=1}^{K_{u}}\left(p_{l}\right)^{w_{l}} \cdot(1)^{w_{K_{u}+1}}
$$

Proof: If $\mathcal{I}_{Y}$ is log-convex then $Y$ must be log-convex as well. Conversely, we need to prove that $\mathcal{I}_{Y}$ is log-convex. Consider two arbitrary $\hat{\boldsymbol{p}}, \check{\boldsymbol{p}} \in \mathbb{R}_{++}^{K_{u}+1}$. We define

$$
\begin{aligned}
& \underline{\boldsymbol{s}}(\lambda)=(1-\lambda) \underline{\hat{\boldsymbol{s}}}+\lambda \underline{\check{\boldsymbol{s}}}, \quad \lambda \in(0,1) \\
& \underline{\boldsymbol{p}}(\lambda)=\exp \underline{\boldsymbol{s}}(\lambda)=\underline{\hat{\boldsymbol{p}}}^{(1-\lambda)} \cdot \underline{\check{\boldsymbol{p}}}^{\lambda} .
\end{aligned}
$$

Because $Y$ is log-convex by assumption, we have

$$
\begin{aligned}
\mathcal{I}_{Y}(\underline{\boldsymbol{p}}(\lambda)) & \underline{\hat{p}}_{K_{u}+1}^{(1-\lambda)} \cdot \underline{\check{p}}_{K_{u}+1}^{\lambda} \\
& \times Y\left(\exp \left[\left(\hat{s}_{1}-\hat{s}_{K_{u}+1}\right)(1-\lambda)+\left(\check{s}_{1}-\check{s}_{K_{u}+1}\right) \lambda\right]\right. \\
& \left.\ldots, \exp \left[\left(\hat{s}_{K_{u}}-\hat{s}_{K_{u}+1}\right)(1-\lambda)+\left(\check{s}_{K_{u}}-\check{s}_{K_{u}+1}\right) \lambda\right]\right) \\
\leq & \exp \left[\underline{\hat{s}}_{K_{u}+1}(1-\lambda)+\underline{\underline{s}}_{K_{u}+1} \lambda\right] \\
& \times Y\left(\exp \left(\hat{s}_{1}-\hat{s}_{K_{u}+1}\right), \ldots, \exp \left(\hat{s}_{K_{u}}-\hat{s}_{K_{u}+1}\right)\right)^{1-\lambda} \\
& \times Y\left(\exp \left(\check{s}_{1}-\check{s}_{K_{u}+1}\right), \ldots, \exp \left(\check{s}_{K_{u}}-\check{s}_{K_{u}+1}\right)\right)^{\lambda} \\
= & \left(\mathcal{I}_{Y}(\underline{\hat{\boldsymbol{p}}})\right)^{1-\lambda} \cdot\left(\mathcal{I}_{Y}(\underline{\underline{\boldsymbol{p}}})\right)^{\lambda} .
\end{aligned}
$$

Thus, $\log \mathcal{I}_{Y}(\exp \underline{\boldsymbol{s}})$ is convex on $\mathbb{R}^{K_{u}+1}$.

\section{B. Characterization of Standard Interference Functions}

Consider Theorem 14, which shows that a weakly standard interference function $Y$ is convex if and only if there exists a set $\mathcal{V}$ such that (64) is fulfilled. In this section we show corresponding results for the case that the function is standard instead of weakly standard.

Theorem 17: $Y$ is a convex standard interference function if and only if for any $\boldsymbol{p}>0$ the optimization problem (64) has a maximizer $\hat{\boldsymbol{v}}=\hat{\boldsymbol{v}}(\boldsymbol{p}) \in \mathcal{V}$ such that $\hat{v}_{K_{u}+1}>0$.

Proof: Suppose that such a maximizer always exists, then for any $\boldsymbol{p}>0$ and any $\lambda>1$ we have

$$
\begin{aligned}
Y(\lambda \boldsymbol{p}) & =\sum_{k=1}^{K_{u}} \hat{v}_{k}(\lambda) \cdot \lambda p_{k}+\hat{v}_{K_{u}+1}(\lambda) \\
& =\lambda\left(\sum_{k=1}^{K_{u}} \hat{v}_{k}(\lambda) p_{k}+\frac{\hat{v}_{K_{u}+1}(\lambda)}{\lambda}\right) \\
& <\lambda\left(\sum_{k=1}^{K_{u}} \hat{v}_{k}(\lambda) p_{k}+\hat{v}_{K_{u}+1}(\lambda)\right) \\
& \leq \lambda \max _{\boldsymbol{v} \in \mathcal{V}}\left(\sum_{k=1}^{K_{u}} v_{k} p_{k}+v_{K_{u}+1}\right)=\lambda \cdot Y(\boldsymbol{p}) .
\end{aligned}
$$

That is, $Y$ is a standard interference function.
Conversely, assume that $Y$ is a standard interference function, and there is a $\boldsymbol{p}>0$ such that for all maximizers $\hat{\boldsymbol{v}}$ (which is a convex subset of $\mathcal{V}$ ) we have $\hat{v}_{K_{u}+1}=0$. Then

$$
\begin{aligned}
\mathcal{I}_{Y}(\boldsymbol{p}, 1) & =Y(\boldsymbol{p}) \\
& =\max _{\boldsymbol{v} \in \mathcal{V}}\left(\sum_{k=1}^{K_{u}} v_{k} p_{k}+v_{K_{u}+1}\right)=\sum_{k=1}^{K_{u}} \hat{v}_{k} p_{k} .
\end{aligned}
$$

For $0<\mu<1$ we have

$$
\begin{aligned}
\mathcal{I}_{Y}(\boldsymbol{p}, \mu) & =\max _{\boldsymbol{v} \in \mathcal{V}}\left(\sum_{k=1}^{K_{u}} v_{k} p_{k}+\mu v_{K_{u}+1}\right) \\
& \geq \sum_{k=1}^{K_{u}} \hat{v}_{k} p_{k}+\mu \hat{v}_{K_{u}+1} \\
& =\sum_{k=1}^{K_{u}} \hat{v}_{k} p_{k}=\mathcal{I}_{Y}(\boldsymbol{p}, 1) .
\end{aligned}
$$

Thus, $\mathcal{I}_{Y}(\boldsymbol{p}, \mu)=\mathcal{I}_{Y}(\boldsymbol{p}, 1)$ for all $0<\mu<1$. Consider an arbitrary $\lambda>1$. We have

$$
\begin{aligned}
Y(\lambda \boldsymbol{p}) & =\mathcal{I}_{Y}(\lambda \boldsymbol{p}, 1) \\
& =\lambda \mathcal{I}_{Y}\left(\boldsymbol{p}, \frac{1}{\lambda}\right)=\lambda \mathcal{I}_{Y}(\boldsymbol{p}, 1)=\lambda Y(\boldsymbol{p}) .
\end{aligned}
$$

Thus, $Y$ is not a standard interference function, which is a contradiction. Thus, if $Y$ is a standard interference function then for any $\boldsymbol{p}>0$ there is an optimizer $\hat{v}=\hat{v}(\boldsymbol{p})$ such that $\hat{v}_{K_{u}+1}>0$.

In a similar way, the corresponding result is shown for concave standard interference functions.

Theorem 18: $Y$ is a concave standard interference function if and only if for any $\boldsymbol{p}>0$ problem (73) has a minimizer $\hat{\boldsymbol{v}}=\hat{\boldsymbol{v}}(\boldsymbol{p}) \in \mathcal{V}$ such that $\hat{v}_{K_{u}+1}>0$.

Finally, we study the case of log-convex standard interference functions.

Theorem 19: $Y$ is a log-convex standard interference function if and only if for any $\boldsymbol{p}>0$ problem (76) has a maximizer $\hat{\boldsymbol{w}}=\hat{\boldsymbol{w}}(\boldsymbol{p})$ such that $\hat{w}_{K_{u}+1}>0$.

Proof: Assume that $Y$ is a log-convex standard interference function, and there is a $\boldsymbol{p}>0$ such that for all maximizers $\hat{\boldsymbol{w}}=\hat{\boldsymbol{w}}(\boldsymbol{p})$ we always have $\hat{w}_{K_{u}+1}(\boldsymbol{p})=0$. For all $\lambda>0$ we have $Y(\lambda \boldsymbol{p}) \leq \lambda Y(\boldsymbol{p})$. Exploiting $\sum_{l=1}^{K_{u}} \hat{w}_{l}=1$, we have

$$
\begin{aligned}
\lambda Y(\boldsymbol{p}) & =\lambda f_{\mathcal{I}}(\hat{\boldsymbol{w}}) \prod_{l=1}^{K_{u}}\left(p_{l}\right)^{\hat{w}_{l}} \\
& =\lambda \sum_{l=1}^{K_{u}} \hat{w}_{l} \cdot f_{\mathcal{I}}(\hat{\boldsymbol{w}}) \prod_{l=1}^{K_{u}}\left(p_{l}\right)^{\hat{w}_{l}} \\
& =f_{\mathcal{I}}(\hat{\boldsymbol{w}}) \prod_{l=1}^{K_{u}}\left(\lambda p_{l}\right)^{\hat{w}_{l}} \leq \max _{\boldsymbol{w}} f_{\mathcal{I}}(\boldsymbol{w}) \prod_{l=1}^{K_{u}}\left(\lambda p_{l}\right)^{w_{l}} \\
& =Y(\lambda \boldsymbol{p}) .
\end{aligned}
$$

Thus, $Y$ is not a standard interference function. This contradiction shows that $\hat{w}_{K_{u}+1}>0$.

Conversely, assume that for any $\boldsymbol{p}>0$ there is always a maximizer $\hat{\boldsymbol{w}}=\hat{\boldsymbol{w}}(\boldsymbol{p})$ such that $\hat{w}_{K_{u}+1}>0$. For a given $\boldsymbol{p}$, we 
study $\lambda \boldsymbol{p}$, where $\lambda>1$. The maximizer is $\hat{\boldsymbol{w}}=\hat{\boldsymbol{w}}(\lambda \boldsymbol{p})$, with $\hat{w}_{K_{u}+1}>0$. With $\sum_{l=1}^{K_{u}} \hat{w}_{l}+\hat{w}_{K_{u}+1}=1$ (see [16]), we have

$$
\begin{aligned}
Y(\lambda \boldsymbol{p}) & =f_{\mathcal{I}}(\hat{\boldsymbol{w}}) \cdot \prod_{l=1}^{K_{u}}\left(\lambda p_{l}\right)^{\hat{\boldsymbol{w}}_{l}} \\
& =(\lambda)^{\sum_{l=1}^{K_{u}} \hat{w}_{l}} \cdot f_{\mathcal{I}}(\hat{\boldsymbol{w}}) \cdot \prod_{l=1}^{K_{u}}\left(p_{l}\right)^{\hat{\boldsymbol{w}}_{l}} \\
& \leq \frac{\lambda}{\lambda^{\hat{w}_{K_{u}+1}}} \cdot \max _{\boldsymbol{w}} f_{\mathcal{I}}(\boldsymbol{w}) \prod_{l=1}^{K_{u}}\left(p_{l}\right)^{\boldsymbol{w}_{l}} \\
& =\frac{\lambda}{\lambda^{\hat{w}_{K_{u}+1}}} \cdot Y(\boldsymbol{p})<\lambda Y(\boldsymbol{p}),
\end{aligned}
$$

because $\lambda>1$ and $\hat{w}_{K_{u}+1}>0$. Thus, $Y$ is standard.

\section{CONCLUSION}

Convexity and monotonicity are two important design principles, which have proven useful for the development of algorithms for joint interference reduction and resource allocation.

- Monotonicity was exploited in the context of axiomatic standard interference functions [9], where a globally convergent fixed point iteration was derived.

- Convexity is widely used, e.g., in the context of multiuser beamforming and robust signal processing. It was also analyzed within the more general axiomatic framework of scale-invariant interference functions [14].

Most existing research is focused on either the first or the second case. In this paper we propose a unifying framework that provides a bridge, allowing to choose the best of both worlds.

The paper shows that certain key properties are preserved when changing from one axiomatic framework to another. Thus, for any given problem we can use the model which is most appropriate. All the structure results shown in [15], [16], and [22] can be transfered to standard interference functions. Conversely, strict monotonicity can be included in the framework A1, A2, A3.

This opens up new analytical possibilities for the design of algorithms for joint transceiver optimization and resource allocation. An example was already given in this paper: By exploiting strict monotonicity, we have shown that the SINR balancing problem has an equivalent convex formulation if the underlying interference functions are convex, concave, or log-convex. This extends the existing literature, where such reformulations are only known for special cases, like the multiuser beamforming problem. The chosen axiomatic approach generalizes this observation to a broader class of resource allocation problems.

\section{APPENDIX I}

\section{A. Beamforming Example}

Consider an uplink system with $K_{u}$ single-antenna transmitters and an $M$-element antenna array at the receiver. Independent signals $s_{1}, \ldots, s_{K_{u}}$ are transmitted over vector-valued channels $\boldsymbol{h}_{1}, \ldots, \boldsymbol{h}_{K_{u}} \in \mathbb{C}^{M}$, with spatial covariance matrices $\boldsymbol{R}_{k}=\mathrm{E}\left[\boldsymbol{h}_{k} \boldsymbol{h}_{k}^{H}\right]$. The superimposed signals at the array output are received by a bank of linear filters $\boldsymbol{u}_{1}, \ldots, \boldsymbol{u}_{K_{u}}$ (the 'beamformers'). The output of the $k$ th beamformer is

$$
y_{k}=\boldsymbol{u}_{k}^{H}\left(\sum_{l \in \mathcal{K}_{u}} \boldsymbol{h}_{l} s_{l}+\boldsymbol{n}\right),
$$

where $\boldsymbol{n} \in \mathbb{C}^{M}$ is an AWGN vector, with $\mathrm{E}\left[\boldsymbol{n} \boldsymbol{n}^{H}\right]=\sigma^{2} \boldsymbol{I}$. The coupling coefficients of the $k$ th user are

$$
\left[\boldsymbol{v}_{k}\left(\boldsymbol{u}_{k}\right)\right]_{l}= \begin{cases}\frac{\boldsymbol{u}_{k}^{H} \boldsymbol{R}_{l} \boldsymbol{u}_{k}}{\boldsymbol{u}_{k}^{H} \boldsymbol{R}_{k} \boldsymbol{u}_{k}} & 1 \leq l \leq K_{u}, l \neq k \\ \frac{\left\|\boldsymbol{u}_{k}\right\|^{2}}{\boldsymbol{u}_{k}^{H} \boldsymbol{R}_{k} \boldsymbol{u}_{k}} & l=K_{u}+1 \\ 0 & l=k .\end{cases}
$$

With the commonly used normalization $\left\|\boldsymbol{u}_{k}\right\|_{2}=1$, the interference function for the beamforming case is

$$
\begin{aligned}
\mathcal{I}_{k}(\underline{\boldsymbol{p}}) & =\left[\max _{\left\|\boldsymbol{u}_{k}\right\|_{2}=1} \frac{\boldsymbol{u}_{k}^{H} \boldsymbol{R}_{k} \boldsymbol{u}_{k}}{\boldsymbol{u}_{k}^{H}\left(\sum_{l \neq k} p_{l} \boldsymbol{R}_{l}+\sigma^{2} \boldsymbol{I}\right) \boldsymbol{u}_{k}}\right]^{-1} \\
& =\min _{\left\|\boldsymbol{u}_{k}\right\|_{2}=1} \underline{\boldsymbol{p}}^{T} \boldsymbol{v}_{k}\left(\boldsymbol{u}_{k}\right) .
\end{aligned}
$$

It can be observed that the interference coupling is not constant. For any power vector $\boldsymbol{p}>0$, the beamformer $\boldsymbol{u}_{k}$ adapts to the interference in such a way that the signal-to-interferenceplus-noise ratio (SINR) is maximized. This optimization can be solved efficiently via an eigenvalue decomposition. For deterministic channels $\boldsymbol{h}_{1}, \ldots, \boldsymbol{h}_{K_{u}}$, we have $\boldsymbol{R}_{\boldsymbol{l}}=\boldsymbol{h}_{l} \boldsymbol{h}_{\boldsymbol{l}}^{H}$, so the interference resulting from optimum beamformers is obtained in closed form

$$
\mathcal{I}_{k}(\underline{\boldsymbol{p}})=\frac{1}{\boldsymbol{h}_{k}^{H}\left(\sigma^{2} \boldsymbol{I}+\sum_{l \neq k} p_{l} \boldsymbol{h}_{l} \boldsymbol{h}_{l}^{H}\right)^{-1} \boldsymbol{h}_{k}} .
$$

For a downlink scenario, we can exploit the reciprocity between uplink and downlink channels. So optimal downlink beamformers can be found indirectly via a "virtual uplink" channel [12], [13].

\section{B. Continuity on the Boundary-Lemmas and Proofs}

We begin by considering an arbitrary vector

$$
\boldsymbol{p}=\left[p_{1}, \ldots, p_{r}, p_{r+1}, \ldots, p_{K}\right]^{T}
$$

where $p_{l}=0$ for $1 \leq l \leq r$ and $p_{l}>0$ for $r+1 \leq l \leq K$. We introduce an arbitrary sequence $\left\{\boldsymbol{\epsilon}^{(n)}\right\}_{n \in \mathbb{N}}$, with

$$
\epsilon^{(n)}=\left[\epsilon_{1}^{(n)}, \ldots, \epsilon_{r}^{(n)}\right]^{T}>0
$$

and $\lim _{n \rightarrow \infty} \boldsymbol{\epsilon}^{(n)}=[0, \ldots, 0]^{T}$. With the nonzero components of $\boldsymbol{p}$, we define

$$
\boldsymbol{p}^{(n)}=\left[\epsilon_{1}^{(n)}, \ldots, \epsilon_{r}^{(n)}, p_{r+1}, \ldots, p_{K}\right]^{T} \in \mathbb{R}_{++}^{K} .
$$

Note, that there are many possible choices of null sequences $\boldsymbol{\epsilon}^{(n)}$. They all converge to the same limit $\boldsymbol{p}=\lim _{n \rightarrow \infty} \boldsymbol{p}^{(n)}$. 
The first lemma shows that the limit of the resulting interference function is always the same, irrespective of the choice of $\boldsymbol{p}^{(n)}$.

Lemma 5: Consider an arbitrary interference function $\mathcal{I}$ defined on $\mathbb{R}_{++}^{K}$. For any $\boldsymbol{p} \in \mathbb{R}_{+}^{K}$ there is a value $\mathcal{I}^{c}(\boldsymbol{p})=$ $\mathcal{I}^{c}\left(p_{r+1}, \ldots, p_{K}\right)$ such that for all possible positive sequences $\left\{\epsilon_{1}^{(n)}\right\}, \ldots,\left\{\epsilon_{r}^{(n)}\right\}, n \in \mathbb{N}$, with $\boldsymbol{p}=\lim _{n \rightarrow \infty} \boldsymbol{p}^{(n)}$, we have

$$
\lim _{n \rightarrow \infty} \mathcal{I}\left(\boldsymbol{p}^{(n)}\right)=\mathcal{I}^{c}(\boldsymbol{p}) .
$$

Proof: We define

$$
\begin{aligned}
\bar{\epsilon}^{(n)} & =\max _{1 \leq k \leq K} \epsilon_{k}^{(n)} \\
\underline{\epsilon}^{(n)} & =\min _{1 \leq k \leq K} \epsilon_{k}^{(n)} .
\end{aligned}
$$

For all $n \in \mathbb{N}$ we have $\bar{\epsilon}^{(n)} \geq \underline{\epsilon}^{(n)}>0$. With

$$
\begin{aligned}
& \overline{\boldsymbol{p}}^{(n)}=\left[\bar{\epsilon}^{(n)}, \ldots, \bar{\epsilon}^{(n)}, p_{r+1}, \ldots, p_{K}\right]^{T} \\
& \underline{\boldsymbol{p}}^{(n)}=\left[\underline{\epsilon}^{(n)}, \ldots, \underline{\epsilon}^{(n)}, p_{r+1}, \ldots, p_{K}\right]^{T}
\end{aligned}
$$

we have $\underline{\boldsymbol{p}}^{(n)} \leq \boldsymbol{p}^{(n)} \leq \overline{\boldsymbol{p}}^{(n)}$, thus $\mathcal{I}\left(\underline{\boldsymbol{p}}^{(n)}\right) \leq \mathcal{I}\left(\boldsymbol{p}^{(n)}\right) \leq$ $\mathcal{I}\left(\overline{\boldsymbol{p}}^{(n)}\right)$. There exist limits

$$
\begin{aligned}
\bar{C}_{1} & =\limsup _{n \rightarrow \infty} \mathcal{I}\left(\overline{\boldsymbol{p}}^{(n)}\right) \\
\underline{C}_{1} & =\liminf _{n \rightarrow \infty} \mathcal{I}\left(\underline{\boldsymbol{p}}^{(n)}\right) .
\end{aligned}
$$

We have

$$
\underline{C}_{1} \leq \liminf _{n \rightarrow \infty} \mathcal{I}\left(\boldsymbol{p}^{(n)}\right) \leq \limsup _{n \rightarrow \infty} \mathcal{I}\left(\boldsymbol{p}^{(n)}\right) \leq \bar{C}_{1}
$$

Next, we show that this is fulfilled with equality. Consider an arbitrary $v \in \mathbb{N}$. By assumption we have $\lim _{n \rightarrow \infty} \bar{\epsilon}^{(n)}=$ $\lim _{n \rightarrow \infty} \underline{\epsilon}^{(n)}=0$, thus there exists an $n_{0}=n_{0}(v)$ such that $\bar{\epsilon}^{(n)} \leq \underline{\epsilon}^{(v)}$ for all $n \geq n_{0}$. Thus, $\overline{\boldsymbol{p}}^{(n)} \leq \boldsymbol{p}^{(v)}$, and with A3 we have $\mathcal{I}\left(\overline{\boldsymbol{p}}^{(n)}\right) \leq \mathcal{I}\left(\underline{\boldsymbol{p}}^{(v)}\right)$, implying $\bar{C}_{1} \leq \mathcal{I}\left(\underline{\boldsymbol{p}}^{(v)}\right)$. This inequality holds for any $\bar{v} \in \mathbb{N}$, thus

$$
\bar{C}_{1} \leq \liminf _{v \rightarrow \infty} \mathcal{I}\left(\underline{\boldsymbol{p}}^{(v)}\right)=\underline{C}_{1} .
$$

Combining (90) with (89) we have $\bar{C}_{1}=\underline{C}_{1}$. From (89) we know that there exists $\mathcal{I}^{c}=\lim _{n} \rightarrow \infty \mathcal{I}\left(\boldsymbol{p}^{(n)}\right)$. This limit does not depend on the choice of the null sequences.

Based on Lemma 5 we can show scale invariance (A2) on the boundary:

Lemma 6: Consider an arbitrary interference function $\mathcal{I}$ defined on $\mathbb{R}_{++}^{K}$. Let $\boldsymbol{p} \in \mathbb{R}_{+}^{K}$. For all $\lambda>0$ we have

$$
\mathcal{I}^{c}(\lambda \boldsymbol{p})=\lambda \mathcal{I}^{c}(\boldsymbol{p})
$$

Proof: For any $\boldsymbol{p}^{(n)}>0$ we have $\mathcal{I}\left(\lambda \boldsymbol{p}^{(n)}\right)=\lambda \mathcal{I}\left(\boldsymbol{p}^{(n)}\right)$. The result follows from (88) and $\lim _{n \rightarrow \infty} \mathcal{I}\left(\alpha \boldsymbol{p}^{(n)}\right)=$ $\mathcal{I}^{c}(\alpha \boldsymbol{p})$.

Also based on Lemma 5 we can prove the following Lemma 7 , which shows monotonicity under the restrictive assumption that the compared vectors have zero entries at the same positions.

Lemma 7: Consider an arbitrary interference function $\mathcal{I}$ defined on $\mathbb{R}_{++}^{K}$. Let $\hat{\boldsymbol{p}}$ and $\check{\boldsymbol{p}}$ be two arbitrary vectors from $\mathbb{R}_{+}^{K}$ with $\hat{p}_{l}=\check{p}_{l}=0$ for $1 \leq l \leq r$ and $\hat{p}_{l} \geq \check{p}_{l}>0$ for $r+1 \leq l \leq K$. Then

$$
\mathcal{I}^{c}(\hat{\boldsymbol{p}}) \geq \mathcal{I}^{c}(\check{\boldsymbol{p}})
$$

Proof: Let $\epsilon^{(n)}>0$ be an arbitrary null sequence, and

$$
\begin{aligned}
& \hat{\boldsymbol{p}}^{(n)}=\left[\epsilon^{(n)}, \ldots, \epsilon^{(n)}, \hat{p}_{r+1}, \ldots, \hat{p}_{K}\right]^{T} \\
& \check{\boldsymbol{p}}^{(n)}=\left[\epsilon^{(n)}, \ldots, \epsilon^{(n)}, \check{p}_{r+1}, \ldots, \check{p}_{K}\right]^{T} .
\end{aligned}
$$

From Lemma 5 we know that $\lim _{n} \rightarrow \infty \mathcal{I}\left(\hat{\boldsymbol{p}}^{(n)}\right)=\mathcal{I}^{c}(\hat{\boldsymbol{p}})$ and $\lim _{n \rightarrow \infty} \mathcal{I}\left(\check{\boldsymbol{p}}^{(n)}\right)=\mathcal{I}^{c}(\check{\boldsymbol{p}})$. Inequality $\mathcal{I}\left(\hat{\boldsymbol{p}}^{(n)}\right) \geq \mathcal{I}\left(\check{\boldsymbol{p}}^{(n)}\right)$ implies (92).

Note, that Lemma 7 does not show monotonicity for arbitrary $\hat{\boldsymbol{p}} \geq \hat{\boldsymbol{p}}$. This is because Lemma 5 and Lemma 7 assume that the positions of the nonzero entries are fixed. So in order to show A3 we need to extend the results to the case of an arbitrary sequence $\left\{\boldsymbol{p}^{(n)}\right\}>0$. Based on the previous Lemmas 5, 6, and 7, we show the following result. It extends Lemma 5 to the case of arbitrary sequences from $\mathbb{R}_{++}^{K}$. This provides a basis for Theorem 5, where general continuity of $\mathcal{I}^{c}$ is shown.

Lemma 8: Let $\boldsymbol{p} \in \mathbb{R}_{+}^{K}$ be arbitrary. For an arbitrary sequence $\boldsymbol{p}^{(n)}=\left[\epsilon_{1}^{(n)}, \ldots, \epsilon_{K}^{(n)}\right]^{T}$, with $\epsilon_{k}^{(n)} \in \mathbb{R}_{++}$, and $\lim _{n \rightarrow \infty} \boldsymbol{p}^{(n)}=\boldsymbol{p}$, we have

$$
\lim _{n \rightarrow \infty} \mathcal{I}\left(\boldsymbol{p}^{(n)}\right)=\mathcal{I}^{c}(\boldsymbol{p}) .
$$

Proof: Consider $\bar{\delta}^{(n)}=\max _{k}\left|p_{k}^{(n)}-p_{k}\right|$ and $\overline{\boldsymbol{p}}^{(n)}=\boldsymbol{p}+$ $\bar{\delta}^{(n)} \mathbf{1}$, where $\mathbf{1}$ is the all-ones vector. With Lemma 7 we have

$$
\begin{aligned}
& \mathcal{I}\left(\overline{\boldsymbol{p}}^{(n)}\right) \geq \mathcal{I}^{c}(\boldsymbol{p}) \\
& \mathcal{I}\left(\overline{\boldsymbol{p}}^{(n)}\right) \geq \mathcal{I}\left(\boldsymbol{p}^{(n)}\right) .
\end{aligned}
$$

Thus,

$$
\begin{aligned}
& \liminf _{n \rightarrow \infty} \mathcal{I}\left(\overline{\boldsymbol{p}}^{(n)}\right) \geq \mathcal{I}^{c}(\boldsymbol{p}) \\
& \liminf _{n \rightarrow \infty} \mathcal{I}\left(\overline{\boldsymbol{p}}^{(n)}\right) \geq \liminf _{n \rightarrow \infty} \mathcal{I}\left(\boldsymbol{p}^{(n)}\right) \\
& \limsup _{n \rightarrow \infty} \mathcal{I}\left(\overline{\boldsymbol{p}}^{(n)}\right) \geq \limsup _{n \rightarrow \infty} \mathcal{I}\left(\boldsymbol{p}^{(n)}\right) .
\end{aligned}
$$

Consider an arbitrary $\epsilon>0$ and $\mathcal{K}_{+}=\{k \in\{1,2, \ldots, K\}$ : $\left.p_{k}>0\right\}$. There exists a $n_{0}=n_{0}(\epsilon)$ such that for all $n \geq n_{0}$ we have $\bar{\delta}^{(n)} \leq \epsilon$. We define

$$
\left[\mathbf{1}_{\mathcal{K}_{+}}\right]_{k}= \begin{cases}1 & k \in \mathcal{K}_{+} \\ 0 & k \notin \mathcal{K}_{+} .\end{cases}
$$

The complement of $\mathcal{K}_{+}$is $\mathcal{K}_{+}^{c}=\mathcal{K} \backslash \mathcal{K}_{+}$. For all $n \geq n_{0}$ we have

$$
\boldsymbol{p} \leq \boldsymbol{p}+\bar{\delta}^{(n)} \mathbf{1} \leq \boldsymbol{p}+\epsilon \mathbf{1}_{\mathcal{K}_{+}}+\bar{\delta}^{(n)} \mathbf{1}_{\mathcal{K}_{+}^{c}},
$$

and thus

$$
\mathcal{I}^{c}(\boldsymbol{p}) \leq \mathcal{I}\left(\boldsymbol{p}+\bar{\delta}^{(n)} \mathbf{1}\right) \leq \mathcal{I}\left(\boldsymbol{p}+\epsilon \mathbf{1}_{\mathcal{K}_{+}}+\bar{\delta}^{(n)} \mathbf{1}_{\mathcal{K}_{+}^{c}}\right)
$$

With Lemma 5 we have

$$
\lim _{n \rightarrow \infty} \mathcal{I}\left(\boldsymbol{p}+\epsilon \mathbf{1}_{\mathcal{K}_{+}}+\bar{\delta}^{(n)} \mathbf{1}_{\mathcal{K}_{+}^{c}}\right)=\mathcal{I}\left(\boldsymbol{p}+\epsilon \mathbf{1}_{\mathcal{K}_{+}}\right) .
$$


Combining (100) and (101) yields

$$
\mathcal{I}^{c}(\boldsymbol{p}) \leq \limsup _{n \rightarrow \infty} \mathcal{I}\left(\boldsymbol{p}+\bar{\delta}^{(n)} \mathbf{1}\right) \leq \mathcal{I}\left(\boldsymbol{p}+\epsilon \mathbf{1}_{\mathcal{K}_{+}}\right) .
$$

The function $\mathcal{I}^{c}$ is an interference function (Lemmas 6 and Lemmas 7) . It is thus continuous with respect to components from $\mathcal{K}_{+}$. Relation (102) holds for all $\epsilon>0$, thus letting $\epsilon \rightarrow 0$, we know that (102) can only be fulfilled with equality. We thus have

$$
\mathcal{I}^{c}(\boldsymbol{p})=\limsup _{n \rightarrow \infty} \mathcal{I}\left(\overline{\boldsymbol{p}}^{(n)}\right) .
$$

By definition, $\overline{\boldsymbol{p}}^{(n)}=\boldsymbol{p}+\bar{\delta}^{(n)} 1$, thus $\mathcal{I}^{c}(\boldsymbol{p})=$ $\lim _{n \rightarrow \infty} \mathcal{I}\left(\overline{\boldsymbol{p}}^{(n)}\right)$. Combining (98) and (103) yields

$$
\mathcal{I}^{c}(\boldsymbol{p}) \geq \limsup _{n \rightarrow \infty} \mathcal{I}\left(\boldsymbol{p}^{(n)}\right) .
$$

Now, consider the vector $\tilde{\boldsymbol{p}}^{(n)}$, defined as

$$
\left[\tilde{\boldsymbol{p}}^{(n)}\right]_{k}= \begin{cases}p_{k}^{(n)} & k \in \mathcal{K}_{+} \\ 0 & k \notin \mathcal{K}_{+}\end{cases}
$$

We have $\lim _{n \rightarrow \infty} \tilde{\boldsymbol{p}}^{(n)}=\boldsymbol{p}$. Again, we exploit that $\mathcal{I}^{c}$ is an interference function, so it is continuous with respect to components from $\mathcal{K}_{+}$. Thus, we have $\lim _{n \rightarrow \infty} \mathcal{I}^{c}\left(\tilde{\boldsymbol{p}}^{(n)}\right)=\mathcal{I}^{c}(\boldsymbol{p})$. So with $\tilde{\boldsymbol{p}}^{(n)} \leq \boldsymbol{p}^{(n)}$ and (104) we obtain

$$
\begin{aligned}
\mathcal{I}^{c}(\boldsymbol{p})=\lim _{n \rightarrow \infty} \mathcal{I}^{c}\left(\tilde{\boldsymbol{p}}^{(n)}\right) & \leq \liminf _{n \rightarrow \infty} \mathcal{I}\left(\boldsymbol{p}^{(n)}\right) \\
& \leq \limsup _{n \rightarrow \infty} \mathcal{I}\left(\boldsymbol{p}^{(n)}\right) \leq \mathcal{I}^{c}(\boldsymbol{p}) .
\end{aligned}
$$

We have $\liminf _{n \rightarrow \infty} \mathcal{I}\left(\boldsymbol{p}^{(n)}\right) \leq \lim _{n \rightarrow \infty} \mathcal{I}\left(\boldsymbol{p}^{(n)}\right) \leq$ $\limsup _{n \rightarrow \infty} \mathcal{I}\left(\boldsymbol{p}^{(n)}\right)$, so the above inequality is fulfilled with equality. That is, $\mathcal{I}^{c}(\boldsymbol{p})=\lim _{n} \rightarrow \infty \mathcal{I}\left(\boldsymbol{p}^{(n)}\right)$.

With Lemma 8 we can prove that monotonicity (A3) holds on the extended domain $\mathbb{R}_{+}^{K}$, which includes the boundary of $\mathbb{R}_{++}^{K}$.

Lemma 9: Consider arbitrary $\hat{\boldsymbol{p}}, \check{\boldsymbol{p}} \in \mathbb{R}_{+}^{K}$, with $\hat{\boldsymbol{p}} \geq \check{\boldsymbol{p}}$. Then

$$
\mathcal{I}(\hat{\boldsymbol{p}}) \geq \mathcal{I}(\check{\boldsymbol{p}}) .
$$

Proof: Exploiting Lemma 8, the proof is similar to the proof of Lemma 7.

\section{Proof of Theorem 7}

With (40) we have $\mathcal{I}(\boldsymbol{p}) \geq \underline{\mathcal{I}}(\boldsymbol{p})$ for all $\boldsymbol{p}>0$. Assume that $\mathcal{I}(\boldsymbol{p})$ is convex. From Lemma 2 we know that there exists a $\overline{\mathcal{V}}$ such that

$$
\begin{aligned}
\underline{I}(\boldsymbol{p}) & =\sup _{\boldsymbol{v}>0}\left(\inf _{\boldsymbol{q}>0}\left(\frac{\max _{\overline{\boldsymbol{v}} \in \overline{\mathcal{V}}} \sum_{l \in \mathcal{K}} \bar{v}_{l} q_{l}}{\sum_{l \in \mathcal{K}} v_{l} q_{l}}\right) \sum_{k \in \mathcal{K}} v_{k} p_{k}\right) \\
& \geq \sup _{\boldsymbol{v}>0}\left(\max _{\overline{\boldsymbol{v}} \in \overline{\mathcal{V}}}\left(\inf _{\boldsymbol{q}>0} \frac{\sum_{l \in \mathcal{K}} \bar{v}_{l} q_{l}}{\sum_{l \in \mathcal{K}} v_{l} q_{l}}\right) \sum_{k \in \mathcal{K}} v_{k} p_{k}\right)
\end{aligned}
$$

$$
\begin{aligned}
& =\sup _{\boldsymbol{v}>0}\left(\max _{\overline{\boldsymbol{v}} \in \overline{\mathcal{V}}}\left(\min _{l \in \mathcal{K}} \frac{\bar{v}_{l}}{v_{l}}\right) \sum_{k \in \mathcal{K}} v_{k} p_{k}\right) \\
& =\sup _{\overline{\boldsymbol{v}} \in \overline{\mathcal{V}}}\left(\max _{\boldsymbol{v}>0}\left(\min _{l \in \mathcal{K}} \frac{\bar{v}_{l}}{v_{l}}\right) \sum_{k \in \mathcal{K}} v_{k} p_{k}\right) \\
& \geq \sup _{\overline{\boldsymbol{v}} \in \overline{\mathcal{V}}}\left(\left(\min _{l \in \mathcal{K}} \frac{\bar{v}_{l}}{\bar{v}_{l}}\right) \sum_{k \in \mathcal{K}} \bar{v}_{k} p_{k}\right) \\
& =\sup _{\overline{\boldsymbol{v}} \in \overline{\mathcal{V}}}\left(\sum_{k \in \mathcal{K}} \bar{v}_{k} p_{k}\right)=\mathcal{I}(\boldsymbol{p}) .
\end{aligned}
$$

Thus, $\mathcal{I}(\boldsymbol{p})=\underline{\mathcal{I}}(\boldsymbol{p})$ for all $\boldsymbol{p}>0$.

Conversely, assume that $\mathcal{I}(\boldsymbol{p})=\underline{\mathcal{I}}(\boldsymbol{p})$ holds. Because $\underline{\mathcal{I}}(\boldsymbol{p})$ is convex, also $\mathcal{I}(p)$ is convex.

\section{Proof of Corollary 2}

Let $\mathcal{I}^{\prime}$ be the greatest convex minorant of $\mathcal{I}$. For all $\boldsymbol{p}>0$, we have $\underline{I}(\boldsymbol{p}) \leq \mathcal{I}^{\prime}(\boldsymbol{p}) \leq \mathcal{I}(\boldsymbol{p})$, and thus

$$
\underline{g}_{\underline{\mathcal{I}}}(\boldsymbol{v}) \leq \underline{g}_{\mathcal{I}^{\prime}}(\boldsymbol{v}) \leq \underline{g}_{\mathcal{I}}(\boldsymbol{v}) \quad \text { for all } \boldsymbol{v}>0 .
$$

Consequently,

$$
\begin{aligned}
\underline{I}(\boldsymbol{p}) & =\sup _{\substack{\boldsymbol{v}>0 \\
\|\boldsymbol{v}\|_{1}=1}} g_{\mathcal{I}}(\boldsymbol{v}) \sum_{k \in \mathcal{K}} v_{k} p_{k} \\
\geq & \sup _{\substack{\boldsymbol{v}>0 \\
\|\boldsymbol{v}\|_{1}=1}} g_{\mathcal{I}^{\prime}}(\boldsymbol{v}) \sum_{k \in \mathcal{K}} v_{k} p_{k}=\mathcal{I}^{\prime}(\boldsymbol{p}) .
\end{aligned}
$$

Thus, $\underline{I}(\boldsymbol{p})=\mathcal{I}^{\prime}(\boldsymbol{p})$ for all $\boldsymbol{p}>0$.

\section{REFERENCES}

[1] S. Koskie and Z. Gajic, "SIR-based power control algorithms for wireless CDMA networks: An overview," Dyn. Continuous Discrete and Impulsive Syst. B: Appl. Algorithms, vol. 10-S, pp. 286-293, 2003.

[2] Z.-Q. Luo, T. N. Davidson, G. B. Giannakis, and K. Wong, "Transceiver optimization for block-based multiple access through ISI channels," IEEE Trans. Signal Process., vol. 52, pp. 1037-1052, Apr. 2004.

[3] Z.-Q. Luo and W. Yu, "An introduction to convex optimization for communications and signal processing," IEEE J. Sel. Areas Commun., vol. 24, pp. 1426-1438, Aug. 2006.

[4] M. Bengtsson and B. Ottersten, Handbook of Antennas in Wireless Communications. Boca Raton, FL: CRC, 2001, ch. 18.

[5] D. Hammarwall, M. Bengtsson, and B. Ottersten, "On downlink beamforming with indefinite shaping constraints," IEEE Trans. Signal Process., vol. 54, pp. 3566-3580, Sep. 2006.

[6] A. Wiesel, Y. C. Eldar, and S. Shamai (Shitz), "Linear precoding via conic optimization for fixed MIMO receivers," IEEE Trans. Signal Process., vol. 54, pp. 161-176, 2006.

[7] M. Biguesh, S. Shahbazpanahi, and A. B. Gershman, "Robust downlink power control in wireless cellular systems," EURASIP J. Wireless Commun. Netw., no. 2, pp. 261-272, 2004.

[8] M. Payaró, A. Pascual-Iserte, and M. A. Lagunas, "Robust power allocation designs for multiuser and multiantenna downlink communication systems through convex optimization," IEEE J. Sel. Areas Commun., vol. 25, no. 7, pp. 1390-1401, Sep. 2007.

[9] R. D. Yates, "A framework for uplink power control in cellular radio systems," IEEE J. Sel. Areas Commun., vol. 13, pp. 1341-1348, Sep. 1995.

[10] C. Huang and R. Yates, "Rate of convergence for minimum power assignment algorithms in cellular radio systems," Baltzer/ACM Wireless Netw., vol. 4, pp. 223-231, 1998. 
[11] K. K. Leung, C. W. Sung, W. S. Wong, and T. Lok, "Convergence theorem for a general class of power-control algorithms," IEEE Trans. Commun., vol. 52, pp. 1566-1574, Sep. 2004.

[12] F. Rashid-Farrokhi, K. J. Liu, and L. Tassiulas, "Transmit beamforming and power control for cellular wireless systems," IEEE J. Sel. Areas Commun., vol. 16, pp. 1437-1449, Oct. 1998.

[13] M. Schubert and H. Boche, "Solution of the multi-user downlink beamforming problem with individual SINR constraints," IEEE Trans. Veh. Technol., vol. 53, pp. 18-28, Jan. 2004.

[14] M. Schubert and H. Boche, "QoS-based resource allocation and transceiver optimization," Found. Trends Commun. Inf. Theory, vol. 2, no. 6, 2005/2006.

[15] H. Boche and M. Schubert, "Concave and convex interference functions-general characterizations and applications," IEEE Trans. Signal Process., vol. 56, pp. 4951-4965, Oct. 2008.

[16] H. Boche and M. Schubert, "A calculus for log-convex interference functions," IEEE Trans. Inf. Theory, vol. 54, pp. 5469-5490, Dec. 2008.

[17] H. Boche and M. Schubert, "A superlinearly and globally convergent algorithm for power control and resource allocation with general interference functions," IEEE/ACM Trans. Netw., vol. 16, no. 2, pp. 383-395, Apr. 2008.

[18] P. Kumar, R. Yates, and J. Holtzman, "Power control based on bit error (BER) measurements," in Proc. IEEE Military Commun. Conf. MILCOM 95, McLean, VA, Nov. 1995, pp. 617-620.

[19] S. Ulukus and R. Yates, "Adaptive power control and mmse interference suppression," ACM Wireless Netw., vol. 4, no. 6, pp. 489-496, 1998.

[20] S. Hanly, "An algorithm for combined cell-site selection and power control to maximize cellular spread spectrum capacity," IEEE J. Sel. Areas Commun., vol. 13, pp. 1332-1340, Sep. 1995.

[21] R. Yates and H. Ching-Yao, "Integrated power control and base station assignment," IEEE Trans. Veh. Technol., vol. 44, no. 3, pp. 638-644, Aug. 1995.

[22] H. Boche and M. Schubert, "The structure of general interference functions and applications," IEEE Trans. Inf. Theory, vol. 54, pp. 4980-4990, Nov. 2008.

[23] H. Boche and M. Schubert, "Nash bargaining and proportional fairness for wireless systems," IEEE/ACM Trans. Netw., vol. 17, no. 5, pp. 1453-1466, Oct. 2009.

[24] M. Schubert and H. Boche, "A unifying theory for uplink and downlink multi-user beamforming," in Proc. IEEE Int. Zurich Semin., Switzerland, Feb. 2002
Holger Boche (M'04-SM'07) received the Dipl.-Ing. and Dr.-Ing. degrees in electrical engineering from the Technische Universität Dresden (TU), Germany, in 1990 and 1994, respectively. In 1992 he graduated in mathematics from the TU Dresden, and in 1998 he received the Dr. rer. nat. degree in pure mathematics from the TU Berlin, Germany.

From 1994 to 1997, he did postgraduate studies in mathematics at the Friedrich-Schiller Universität Jena, Germany. In 1997, he joined the Heinrich-Hertz-Institut (HHI) für Nachrichtentechnik Berlin. He is head of the Broadband Mobile Communication Networks Department, HHI. Since 2002, he has been Full Professor for Mobile Communication Networks at the TU Berlin, Institute for Communications Systems, and since 2003, he has been Director of the Fraunhofer German-Sino Lab for Mobile Communications, Berlin. He was a Visiting Professor with the ETH Zurich, Switzerland, during winter term 2004 and 2006 and with KTH Stockholm during summer term 2005.

Professor Boche received the Research Award "Technische Kommunikation" from the Alcatel SEL Foundation in October 2003, the "Innovation Award" from the Vodafone Foundation in June 2006, Gottfried Wilhelm Leibniz Prize from the Deutsche Forschungsgemeinschaft (German Research Foundation) in 2008. He was a corecipient of the 2006 IEEE Signal Processing Society Best Paper Award and recipient of the 2007 IEEE Signal Processing Society Best Paper Award. He is a member of IEEE Signal Processing Society SPCOM and SPTM Technical Committee. He was elected a member of the German Academy of Sciences (Leopoldina) in 2008.

Martin Schubert (M'06) received the diploma and doctoral degrees in electrical engineering from the Technische Universität (TU), Berlin, Germany, in 1998 and 2002, respectively.

In 1998 he joined the Heinrich-Hertz Institute for Telecommunications (HHI), Berlin, as a Research Assistant. Since 2003, he has been with the Fraunhofer German-Sino Lab for Mobile Communications (MCI), where he is working as a Senior Researcher. He is also Lecturer at the TU Berlin. His current research interests include the optimization of wireless communication networks, with particular emphasis on interference management, resource allocation, and multiantenna signal processing.

Dr. Schubert was a corecipient of the VDE Johann-Philipp-Reis Award in 2007. Since 2009, he has been an Associate Editor of the IEEE TRANSACTIONS OF SignAL PROCESSING. He coauthored the 2007 Best Paper Award of the IEEE Signal Processing Society. 